\title{
LOMBARDI DRAWINGS OF KNOTS AND LINKS
}

\author{
Philipp Kindermann*, Stephen Kobourov ${ }^{\dagger}$ Maarten Löffler ${ }^{\ddagger}$ Martin Nöllenburg \\ André Schulz, and Birgit Vogtenhuber\|
}

ABSTRACT. Knot and link diagrams are projections of one or more 3-dimensional simple closed curves into $\mathbb{R}^{2}$, such that no more than two points project to the same point in $\mathbb{R}^{2}$. These diagrams are drawings of 4-regular plane multigraphs. Knots are typically smooth curves in $\mathbb{R}^{3}$, so their projections should be smooth curves in $\mathbb{R}^{2}$ with good continuity and large crossing angles: exactly the properties of Lombardi graph drawings (defined by circular-arc edges and perfect angular resolution).

We show that several knots do not allow crossing-minimal plane Lombardi drawings. On the other hand, we identify a large class of 4-regular plane multigraphs that do have plane Lombardi drawings. We then study two relaxations of Lombardi drawings and show that every knot admits a crossing-minimal plane 2-Lombardi drawing (where edges are composed of two circular arcs). Further, every knot is near-Lombardi, that is, it can be drawn as a plane Lombardi drawing when relaxing the angular resolution requirement by an arbitrary small angular offset $\varepsilon$, while maintaining a $180^{\circ}$ angle between opposite edges.

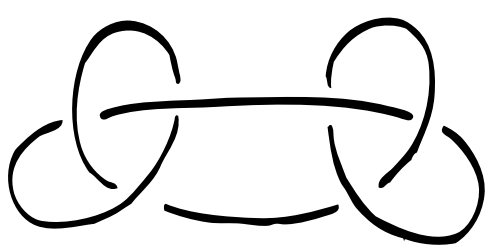

(a)

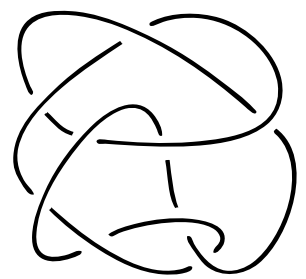

(b)

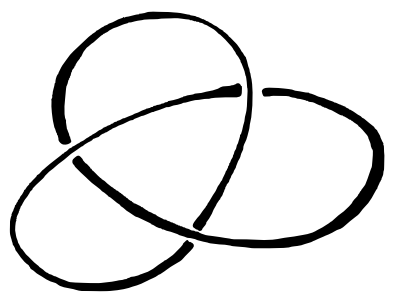

(c)

Figure 1: Hand-made drawings of knots from the books of (a) Rolfsen [19], (b) Livingston [18], and (c) Kauffman [16].

\section{Introduction}

A knot is an embedding of a simple closed curve in 3-dimensional Euclidean space $\mathbb{R}^{3}$. Similarly, a link is an embedding of a collection of simple closed curves in $\mathbb{R}^{3}$. A drawing of

*Universität Würzburg, Germany philipp.kindermann@uni-wuerzburg.de

${ }^{\dagger}$ University of Arizona, Tucson, AZ, US kobourov@cs.arizona.edu

${ }^{\ddagger}$ Universiteit Utrecht, the Netherlands m. loffler@uu.nl

$\S$ TU Wien, Vienna, Austria noellenburg@ac.tuwien.ac.at

"Fern Universität in Hagen, Germany andre.schulz@fernuni-hagen.de

" Graz University of Technology, Austria bvogt@ist.tugraz.at 
a knot (link) (also known as knot diagram) is a projection of the knot (link) to the Euclidean plane $\mathbb{R}^{2}$ such that for any point $p$ of $\mathbb{R}^{2}$, at most two points of the curve(s) are mapped to it $[8,19,20]$; if two points are mapped to $p$, then $p$ is a crossing. A knot diagram of a knot (link) is crossing-minimal if there is no projection of the knot (link) with fewer crossings.

Graph drawing perspective. An embedding of a planar (multi-)graph is a combinatorial description of a planar drawing by listing the cyclic order of the edges on the boundaries of the faces. An embedding uniquely defines a cyclic order of edges around each vertex. A plane (multi-)graph is a planar (multi-)graph together with a combinatorial embedding. Given a knot diagram, we can obtain a plane multigraph by placing a vertex on each crossing. Two vertices are connected if there is a curve in the knot diagram between these two vertices that does not contain any other vertex. Since every vertex corresponds to a crossing, this multigraph is 4-regular. Hence, drawings of knots and links are drawings of 4-regular plane multigraphs. Likewise, every 4-regular plane multigraph can be interpreted as a link. To keep the notation simple, when we talk about a (combinatorial) embedding of a knot, we refer to the (combinatorial) embedding specified by one of its knot diagrams, and not to its embedding in $\mathbb{R}^{3}$. A vertex-minimal embedding of a knot is a (combinatorial) embedding specified by a crossing-minimal knot diagram.

Problem statement. In this paper, we address a question that was recently posed by Benjamin Burton: "Given a drawing of a knot, how can it be redrawn nicely without changing the given topology of the drawing?" We do know what a drawing of a knot is, but what is meant by a nice drawing? Several graphical annotations of knots and links as graphs have been proposed in the knot theory literature, but most of the illustrations are hand-drawn; see Figure 1. When studying these drawings, a few desirable features become apparent: (i) edges are typically drawn as smooth curves, (ii) the angular resolution of the underlying 4-regular graph is close to $90^{\circ}$, and (iii) the drawing preserves the continuity of the knot, that is, in every vertex of the underlying graph, opposite edges have a common tangent. There are many more features one could wish from a drawing of a knot or link, see, e.g., the energy models discussed in the PhD thesis of Scharein [20]. But our task is to redraw a given drawing of a knot with a particular topology, so other typical quality metrics, such as the number of crossings, that vary with the choice of the embedding or topology of a knot diagram do not apply here.

There already exists a graph drawing style that fulfills the three requirements above: a Lombardi drawing of a (multi-)graph $G=(V, E)$ is a drawing of $G$ in the Euclidean plane with the following properties:

1. The vertices are represented as distinct points in the plane

2. The edges are represented as circular arcs connecting the representations of their end vertices (and not containing the representation of any other vertex); note that a straight-line segment is a circular arc with radius infinity.

3. Every vertex has perfect angular resolution, i.e., its incident edges are equiangularly spaced. For knots and links this means that the angle between any two consecutive edges is $90^{\circ}$. 


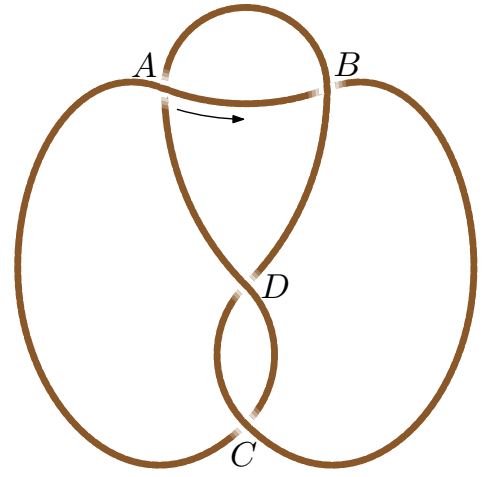

(a) Gauss code: $A,-B, C,-D, B,-A, D,-C$

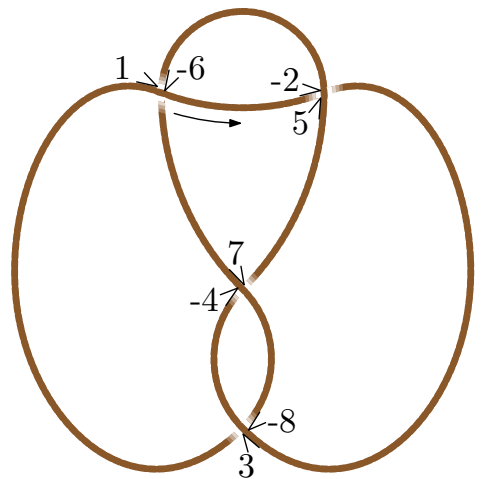

(b) Dowker-Thistlethwaite code: $-6,-8,-2,-4$

Figure 2: Representations of the knot $4_{1}$.

A Lombardi drawing is plane if none of its edges intersect. We are particularly interested in plane Lombardi drawings, since crossings change the topology of the drawn knot. Obviously, if a plane multigraph contains a loop, that is, an edge from a vertex to itself, then it cannot have a Lombardi drawing. Hence, we assume throughout the paper that our multigraphs do not have loops.

Knot diagram representations. There are several ways in the literature to combinatorically represent a knot diagram that are different from the 4-regular multi-graph as described above, which we will briefly survey. The Alexander-Briggs-Rolfsen notation [4,19] is a well established notation that organizes knots by their vertex number and a counting index, e.g., the trefoil knot $3_{1}$ is listed as the first (and only) knot with three vertices. The Gauss code [9] of a knot can be computed as follows. Label each vertex with a letter, then pick a starting vertex and a direction, traverse the knot, and record the labels of the vertices encountered in the order of the traversal with a preceding "-" if the part of the knot that is followed at the vertex lies below the other part (called an under crossing); see Figure 2a. The Dowker-Thistlethwaite code [10] is obtained similar to the Gauss code: Pick a starting vertex and a direction, traverse the knot, and label the vertices in the order of the traversal with consecutive integers, starting from 1, with a preceding "-" in case of an under crossing for even labels. Then, every vertex has two labels: a positive odd label and an even label. Order the vertices ascendingly by their odd label, and record their corresponding even labels in this order; see Figure $2 \mathrm{~b}$.

Knot drawing software. Software for generating drawings for knots and links exists. One powerful package is KnotPlot [20], which provides several methods for drawing knot diagrams. It contains a library of over 1,000 precomputed knots and can also generate knot drawings of certain families, such as torus knots. KnotPlot is mainly concerned with visualizing knots in three and four dimensions. To this end, a knot is represented as a 3 -dimensional path on a set number of nodes, and then forces are used on these nodes to smoothen the visualization without changing the topology. But KnotPlot also provides 
methods for drawing general knots in $2 \mathrm{D}$ based on the embedding of the underlying plane multigraph, represented by the Dowker-Thistlethwaite code. By replacing every vertex by a 4-cycle, the multigraph becomes a simple planar 3-connected graph, which is then drawn using Tutte's barycentric method [23]. In the end, the modifications are reversed and a drawing of the knot is obtained with edges drawn as polygonal arcs. The author noticed that this method "... does not yield "pleasing' graphs or knot diagrams." In particular, he noticed issues with vertex and angular resolution [20, pg. 102].

Another approach was used by Emily Redelmeier [1] in the Mathematica package KnotTheory. Here, every arc, crossing, and face of the knot diagram is associated with a disk. The drawing is then generated from the implied circle packing as a circular arc drawing. As a result of the construction, every edge in the diagram is made of three circular arcs with common tangents at opposite edges. Since no further details are given, it is hard to evaluate the effectiveness of this approach, although as we show in this paper, three circular arcs per edge are never needed. A related drawing style for knots are the so-called arc presentations [7]. An arc presentation is an orthogonal drawing, that is, all edges are sequences of horizontal and vertical segments, with the additional properties that at each vertex the vertical segments are above the horizontal segments in the corresponding knot and that each row and column contains exactly one horizontal and vertical segment, respectively. However, these drawings might require a large number of bends per edge.

Lombardi drawings. Lombardi drawings were introduced by Duncan et al. [12]. They showed that 2-degenerate graphs have Lombardi drawings and that all $d$-regular graphs, with $d \not \equiv 2(\bmod 4)$, have Lombardi drawings with all vertices placed along a common circle. Neither of these results, however, is guaranteed to result in plane drawings. Duncan et al. [12] also showed that there exist planar graphs that do not have plane Lombardi drawings, but restricted graph classes (e.g., Halin graphs) do. In subsequent work, Eppstein [13,14] showed that every (simple) planar graph with maximum degree three has a plane Lombardi drawing. Further, he showed that a certain class of 4-regular planar graphs (the medial graphs of polyhedral graphs) also admit plane Lombardi drawings and he presented an example of a 4-regular planar graph that does not have a plane Lombardi drawing. A generalization of Lombardi drawings are $k$-Lombardi drawings. Here, every edge is a sequence of at most $k$ circular arcs that meet at a common tangent. Duncan et al. [11] showed that every planar graph has a plane 3 -Lombardi drawing. Related to $k$-Lombardi-drawings are smooth-orthogonal drawings of complexity $k$ [5]. These are plane drawings where every edge consists of a sequence of at most $k$ quarter-circles and axis-aligned segments that meet smoothly, edges are axis-aligned (emanate from a vertex either horizontally or vertically), and no two edges emanate in the same direction. Note that in the special case of 4-regular graphs, smooth-orthogonal drawings of complexity $k$ are also plane $k$-Lombardi drawings.

Our Contributions. The main question we study here is motivated by the application of the Lombardi drawing style to knot and link drawings: Given a 4-regular plane multigraph $G$ without loops, does $G$ admit a plane Lombardi drawing with the same combinatorial embedding? In Section 2 we start with some positive results on extending a plane Lombardi drawing, as well as composing two plane Lombardi drawings. In Section 3, by extending the 
results of Eppstein [13,14], we show that a large class of multigraphs, including 4-regular polyhedral graphs, does have plane Lombardi drawings. Unfortunately, there exist several small knots that do not have a vertex-minimal plane Lombardi drawing. Section 4 discusses these cases but also lists a few positive results for small examples. In Section 5, we show that every 4-regular plane multigraph has a plane 2-Lombardi drawing. In Section 6, we show that every 4-regular plane multigraph can be drawn with non-crossing circular arcs, so that the perfect angular resolution criterion is violated only by an arbitrarily small value $\varepsilon$, while maintaining that opposite edges have common tangents.

\section{General Observations}

A plane Lombardi drawing of a knot is a plane Lombardi drawing of one of its (combinatorial) embeddings. A (combinatorial) embedding of a knot is a Lombardi embedding if it admits a plane Lombardi drawing, and a non-Lombardi embedding otherwise. We call the property of admitting a plane Lombardi drawing plane Lombardiness. If two vertices in a plane Lombardi drawing of a knot are connected by a pair of multi-edges that are consecutive in the cyclic order around both vertices specified by the embedding, then we denote the face enclosed by these two edges as a lens. A knot (link) embedding is called reduced if it has no loop or cutvertices. We observe that a vertex-minimal embedding of a knot (link) must be reduced, as otherwise we can flip the order of two edges at such a vertex to remove it and obtain an embedding of the knot (link) with one fewer vertex. In fact, Kauffman, Murasugi, and Thistlethwaite have independently shown that alternating knot (link) diagrams (where "over" and "under" crossings alternate along the curve) are vertex-minimal if and only if they are reduced [2, Chapter 3.3]. Hence, to determine the plane Lombardiness of knots (links), it suffices to consider biconnected multigraphs without loops.

There exist a number of operations that maintain the plane Lombardiness of a 4 regular plane multigraph. Two knots $A$ and $B$ can be combined by connecting $A$ and $B$ along edges $a$ of $A$ and $b$ of $B$, that is, cutting an edge $a$ of $A$ and an edge $b$ of $B$ open and gluing pairwise the loose ends of of $a$ with the loose ends of $b$. This operation is known as a knot sum $A+B$. Knots that cannot be decomposed into a sum of two smaller knots are known as prime knots. By Schubert's theorem, every knot can be uniquely decomposed into prime knots [21]. The smallest prime knot is the trefoil knot with three crossings or vertices; see Figure 1c. Rolfsen's knot table ${ }^{1}$ lists all prime knots with up to ten vertices.

Lemma 1. Let $A$ and $B$ be two 4-regular plane multigraphs with plane Lombardi drawings. Let $a$ be an edge of $A$ and $b$ an edge of $B$. Then the knot sum $A+B$, obtained by connecting $A$ and $B$ along edges $a$ and $b$, admits a plane Lombardi drawing.

Proof. We first apply a Möbius transformation to the plane Lombardi drawings of $A$ and $B$ so that in the resulting drawings the given edges $a$ and $b$ are drawn as straight edges passing through the point at infinity, i.e., they are complements of line segments on an infinite-radius circle; see Figure 3. Next, we rotate and align both of these drawings so that edges $a$ and $b$ are collinear and the subdrawings obtained by removing edges $a$ and $b$ do not intersect. In

${ }^{1}$ http://katlas.org/wiki/The_Rolfsen_Knot_Table 


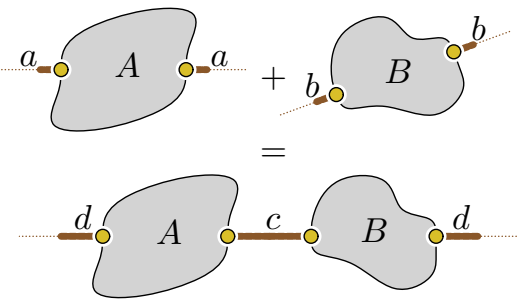

Figure 3: Adding two plane Lombardi drawings of 4-regular multigraphs.

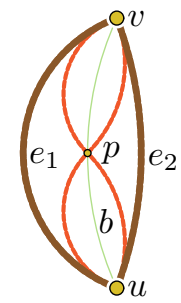

Figure 4: Subdividing a Figure 5: Placement circle lens between $u$ and $v$ by a new vertex $p$.

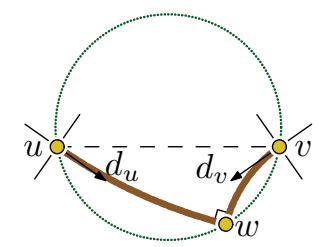

for neighbor $w$ of $u$ and $v$ in a 4-regular graph.

the final step, we remove both $a$ and $b$ and reconnect their vertices by two new edges $c$ and $d$ connecting the two drawings, one being a line segment and the other passing through infinity. Since Möbius transformations preserve planarity and Lombardiness and our construction does not introduce any edge crossings, the resulting drawing is a plane Lombardi drawing. Another Möbius transformation may be applied to make the edge through infinity curved again.

Corollary 2. Let $K$ be a composite knot that can be decomposed into prime knots $A_{1}, \ldots, A_{k}$. If each of $A_{1}, \ldots, A_{k}$ admits a crossing-minimal plane Lombardi drawing, then so does $K$.

We will use the following lemma several times throughout the paper.

Lemma 3 (Property 2 in $[11,12]$ ). Let $u$ and $v$ be two vertices with given positions that have a common, unplaced neighbor $w$. Let $d_{u}$ and $d_{v}$ be two tangent directions and let $\theta$ be a target angle. Let $C$ be the locus of all positions for placing $w$ so that (i) the edge $(u, w)$ is a circular arc leaving $u$ in direction $d_{u}$, (ii) the edge $(v, w)$ is a circular arc leaving $v$ in direction $d_{v}$, and (iii) the angle formed at $w$ is $\theta$. Then $C$ is a circle, the so-called placement circle of $w$.

Duncan et al. [11] further specify the radius and center of the placement circle by the input coordinates and angles. For the special case that the two tangent directions $d_{u}$ and $d_{v}$ are symmetric with respect to the line through $u$ and $v$, and that the angle $\theta$ is $90^{\circ}$ or $270^{\circ}$, the corresponding placement circle is such that its tangent lines at $u$ and $v$ form an angle of $45^{\circ}$ with the arc directions $d_{u}$ and $d_{v}$. In particular, the placement circle bisects the right angle between $d_{u}$ (resp. $d_{v}$ ) and its neighboring arc direction. Figure 5 illustrates this situation.

Another operation that preserves the plane Lombardiness is lens multiplication. Let $G=(V, E)$ be a 4-regular plane multigraph with a lens between two vertices $u$ and $v$. A lens multiplication of $G$ is a 4-regular plane multigraph that is obtained by replacing the lens between $u$ and $v$ with a chain of lenses.

Lemma 4. Let $G=(V, E)$ be a 4-regular plane multigraph with a plane Lombardi drawing $\Gamma$. Then, any lens multiplication $G^{\prime}$ of $G$ also admits a plane Lombardi drawing.

Proof. Let $f$ be a lens in $\Gamma$ spanned by two vertices $u$ and $v$. We denote the two edges bounding the lens as $e_{1}$ and $e_{2}$. If we remove $e_{1}$ and $e_{2}$ from the drawing and add the 
vertex $b$, then the new edges use the same tangents at $u$ and $v$ as $e_{1}$ and $e_{2}$ which are symmetric with respect to the line through $u$ and $v$, and the angle $\theta$ is $90^{\circ}$. Hence, following Duncan et al. [11], the placement circle of $p$ bisects the right angle between $e_{1}$ and $e_{2}$ at $u$ and $v$. We denote by $b$ the part of the placement circle that lies inside $f$; see Figure 4

Let $p$ be the midpoint of $b$. By Lemma 3, if we draw circular arcs from both $u$ and $v$ to $p$ that have the same tangents as $e_{1}$ and $e_{2}$ in $u$ and $v$, then these four arcs meet at $p$ forming angles of $90^{\circ}$. Furthermore, each such arc lies inside lens $f$ and hence does not cross any other arc of $\Gamma$. The resulting drawing is thus a plane Lombardi drawing of a 4-regular multigraph that is derived from $G$ by subdividing the lens $f$ with a new degree- 4 vertex.

By repeating this construction inside the new lenses, we can create plane Lombardi drawings that replace lenses by chains of smaller lenses.

\section{Plane Lombardi Drawings via Circle Packing}

Recall that polyhedral graphs are simple planar 3-connected graphs, and that those graphs have a unique (plane) combinatorial embedding. The (plane) dual graph $M^{\prime}$ of a plane graph $M$ has a vertex for every face of $M$ and an edge between two vertices for every edge shared by the corresponding faces in $M$. In the "classic" drawing $D\left(M, M^{\prime}\right)$ of a primal-dual graph pair $\left(M, M^{\prime}\right)$, every vertex of $M^{\prime}$ lies in its corresponding face of $M$ and vice versa, and every edge of $M^{\prime}$ intersects exactly its corresponding edge of $M$. Hence, every cell of $D\left(M, M^{\prime}\right)$ has exactly two such edge crossings and exactly one vertex of each of $M$ and $M^{\prime}$ on its boundary. The medial graph of a primal-dual graph pair $\left(M, M^{\prime}\right)$ has a vertex for every crossing edge pair in $D\left(M, M^{\prime}\right)$ and an edge between two vertices whenever they share a cell in $D\left(M, M^{\prime}\right)$; see Figure 6a. Every cell of the medial graph contains either a vertex of $M$ or a vertex of $M^{\prime}$ and every edge in the medial graph is incident to exactly one cell in $D\left(M, M^{\prime}\right)$.

Every 4-regular plane multigraph $G$ can be interpreted as the medial graph of some plane graph $M$ and its dual $M^{\prime}$, where both graphs possibly contain multi-edges. In fact, medial graphs have already been used in the context of knot diagrams by Tait in 1879 [22]. If $G$ contains no loops and cutvertices, then neither $M$ nor $M^{\prime}$ contains loops. Eppstein [13] showed that if $M$ (and hence also $M^{\prime}$ ) is polyhedral, then $G$ admits a plane Lombardi drawing.

We first give a high-level overview for Eppstein's algorithm, which uses a primal-dual circle packing. For a plane graph $M$ and its dual $M^{\prime}$, a primal-dual circle packing $C\left(M, M^{\prime}\right)$ consists of two families $C(M)$ and $C\left(M^{\prime}\right)$ of circles, such that there is a bijection between the set of vertices of $M$ and circles of $C(M)$ and a bijection between the set of vertices of $M^{\prime}$ and circles of $C\left(M^{\prime}\right)$. Moreover, the following properties hold:

(1) The circles in the family $C(M)$ are interiorly disjoint and their contact graph is $M$, i.e., two circles touch if and only if there is an edge in $M$ between their corresponding vertices.

(2) If $c(o) \in C\left(M^{\prime}\right)$ is the circle of the outer face $o$, then the circles of $C\left(M^{\prime}\right) \backslash\{c(o)\}$ are interiorly disjoint while $c(o)$ contains all of them. The contact graph of $C\left(M^{\prime}\right)$ is $M^{\prime}$. 
(3) The circle packings $C(M)$ and $C\left(M^{\prime}\right)$ are orthogonal, i.e., if $e=(u, v)$ and the dual of $e$ is $e^{*}=(f, g)$, and $c(u), c(v), c(f), c(g)$ are their respective circles in $C(M)$ and $C\left(M^{\prime}\right)$, then there is a point $p=c(u) \cap c(v)=c(f) \cap c(g)$; moreover, the common tangents $t$ of $c(u), c(v)$ and $t^{*}$ of $c(f), c(g)$ cross perpendicularly in $p_{e}$.

If $M$ and $M^{\prime}$ are polyhedral, then a primal-dual circle packing $C\left(M, M^{\prime}\right)$ always exists due to Brightwell and Schreinerman [6]. As the combinatorial embedding of $M$ and $M^{\prime}$ is unique up to homeomorphism on the sphere, there exists a Möbius transformation $\tau$ such that the circle packing $\tau\left(C\left(M, M^{\prime}\right)\right)$ has the same unbounded face as $D\left(M, M^{\prime}\right)$. Recall that every edge of the medial graph $G$ is incident to exactly one cell in $D\left(M, M^{\prime}\right)$. The corresponding cell in $\tau\left(C\left(M, M^{\prime}\right)\right)$ is the intersection of a primal circle and a dual circle. Eppstein obtains a plane Lombardi drawing of $G$ by placing the vertices on the crossings between the primal and dual circles, and drawing the edges as bisectors of their corresponding cells.

We show next how to extend this result to a larger graph class. In particular, we show that if one of $M$ and $M^{\prime}$ is simple, then $D(G)$ admits a plane Lombardi-drawing. A full construction example of the algorithm can be found in Appendix A.

Theorem 5. Let $G=(V, E)$ be a biconnected 4-regular plane multigraph and let $M$ and $M^{\prime}$ be the primal-dual multigraph pair for which $G$ is the medial graph. If one of $M$ and $M^{\prime}$ is simple, then $G$ admits a plane Lombardi drawing preserving its embedding.

Proof. Assume without loss of generality that $M$ is simple. If $M$ (and hence also $M^{\prime}$ ) is polyhedral, then $G$ admits a plane Lombardi drawing $\Gamma$ by Eppstein [13] as described above.

Now assume that $M$ is not 3-connected. As a first step, we iteratively extend $M=M_{0}$ by adding $p$ edges until we obtain a polyhedral graph $M_{p}$. Since every maximal plane simple graph is polyhedral, this edge addition process does eventually reach a polyhedral graph. During this process, we also iteratively adapt the dual graph and the medial graph; see Figures $6 \mathrm{a}-\mathrm{b}$ for an illustration. Let $M_{i+1}$ be the graph obtained from $M_{i}$ by adding edge $e$ to $M_{i}$. The edge $e$ splits a face $f$ of $M_{i}$ with at least four incident vertices into two faces $f_{1}$ and $f_{2}$ with at least three incident vertices each. In $M_{i}^{\prime}$, the according vertex $f^{\prime}$ is split into two vertices $f_{1}^{\prime}$ and $f_{2}^{\prime}$. The edges incident to $f^{\prime}$ are partitioned into edges incident to $f_{1}^{\prime}$ and $f_{2}^{\prime}$ and an additional edge between $f_{1}^{\prime}$ and $f_{2}^{\prime}$ is added. In $G_{i}$, the edges inside the face $f$ of $M_{i}$ form a cycle that connects every pair of edges in $M_{i}$ that is incident along the boundary of $f$. When $e$ is added, exactly two edges $g_{1}, g_{2}$ of $G_{i}$ are intersected by $e$. To obtain $G_{i+1}$, the edges $g_{1}$ and $g_{2}$ are replaced by four new edges, where each new edge has the new crossing between $e$ and $\left(f_{1}^{\prime}, f_{2}^{\prime}\right)$ as one endpoint and one of the four endpoints of $g_{1}$ and $g_{2}$, respectively, as the other endpoint.

In the second step, we apply the result of Eppstein [13] to obtain a plane Lombardi drawing $\Gamma_{p}$ of $G_{p}$ together with a primal-dual circle packing $C\left(M_{p}, M_{p}^{\prime}\right)$. Before going into the third step, the iterative removal of the edges that were added in the first step, let us consider the structure obtained from the second step in more detail; see Figures $6 \mathrm{c}-6 \mathrm{~d}$. For an edge $g$ of $G_{p}$, consider the unique vertex $m(g) \in M_{p}$ that lies in a cell of $G_{p}$ incident to $g$. Note that $g$ has its endpoints on two edges incident to $m(g)$ and adjacent in their order around $m(g)$. Let these edges be $\left(m(g), m_{1}(g)\right)$ and $\left(m(g), m_{2}(g)\right)$, respectively. 


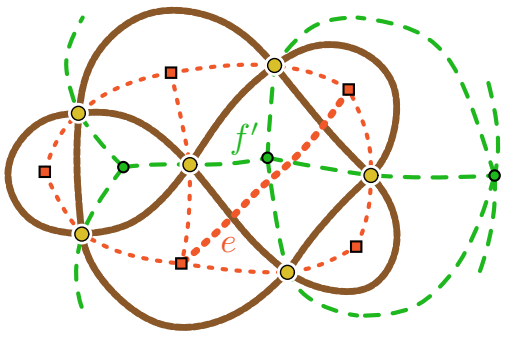

(a)

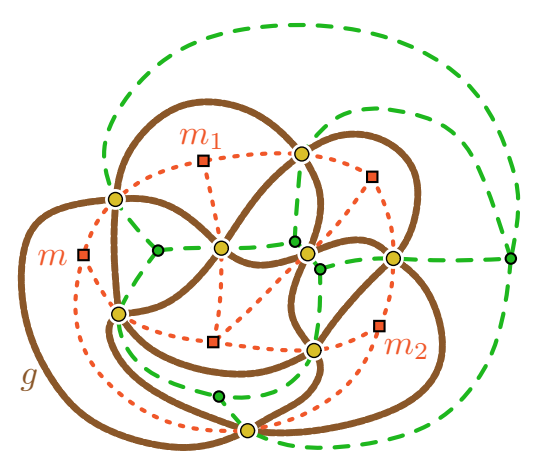

(c)

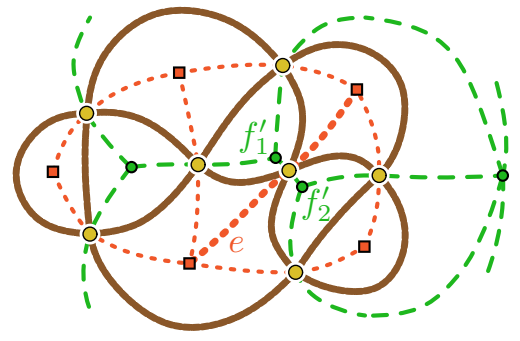

(b)

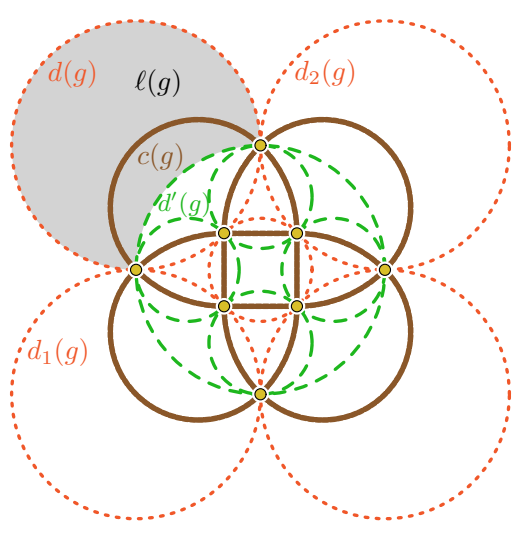

(d)

Figure 6: (a)-(b) Modifications due to the addition of edge $e$. (c) Extension of (b) to a polyhedral graph, and (d) the according primal-dual circle packing representation. The medial graph $G$ is drawn solid, the primal multigraph $M$ is drawn dotted, and the dual multigraph $M^{\prime}$ is drawn dashed. The shaded area is the lens region $l(g)$.

Let $d(g), d_{1}(g)$, and $d_{2}(g)$ be the disks in $C\left(M_{p}\right)$ corresponding to $m(g), m_{1}(g)$, and $m_{2}(g)$, respectively. Then in $\Gamma_{p}$, the circular arc $c(g)$ corresponding to $g$ lies in the interior ${ }^{2}$ of the disk $d(g)$ and has its endpoints on the touching points of $d(g)$ with $d_{1}(g)$ and $d_{2}(g)$, respectively. These touching points are consecutive along the boundary of $d(g)$. Further, there is a disk $d^{\prime}(g)$ in $C\left(M_{p}^{\prime}\right)$ whose boundary intersects the boundary of $d(g)$ exactly in the endpoints of $c(g)$. The intersection of $d(g)$ and $d^{\prime}(g)$ contains $c(g)$ in its interior. The circles $\partial d(g)$ and $\partial d^{\prime}(g)$ intersect with right angles and $c(g)$ bisects the angles at both intersections. We call $d(g) \cap d^{\prime}(g)$ the lens region $\ell(g)$ of $g$. For any two edges $g_{1}$ and $g_{2}$ of $\Gamma_{p}$, the according lens regions $\ell\left(g_{1}\right)$ and $\ell\left(g_{2}\right)$ are interior-disjoint. The lens regions of the edges incident to the face in $\Gamma_{p}$ corresponding to $m(g)$ cover the whole boundary of $d(g)$ and the endpoints of those regions appear in the same cyclic order as the according edges in $M_{p}$.

In the third step, we iteratively remove the edges that were added in the first step, by constructing a sequence of plane Lombardi drawings $\Gamma_{i}$ for $G_{i}$, for $i=p-1, \ldots, 0$. For any edge $g$ of $G_{i}$, consider the unique vertex $m(g) \in M_{i}$ that lies in a cell of $\Gamma_{i}$ incident to $g$, with endpoints on edges $\left(m(g), m_{1}(g)\right)$ and $\left(m(g), m_{2}(g)\right)$ of $M_{i}$, respectively.

\footnotetext{
${ }^{2}$ Here, interior is meant w.r.t. the circle packing. Note that a circle could also be inverted, that is, contain the unbounded face.
} 
Let $d(g), d_{1}(g)$, and $d_{2}(g)$ be the disks in $C\left(M_{p}\right)$ corresponding to $m(g), m_{1}(g)$, and $m_{2}(g)$, respectively, and let $c(g)$ be the circular arc in $\Gamma_{i}$ corresponding to $g$. We keep the following invariants for all edges $g$ of the drawing $\Gamma_{i}$ :

(i) $c(g)$ lies in the disk $d(g)$ and has its endpoints on the touching points of $d(g)$ with $d_{1}(g)$ and $d_{2}(g)$, respectively.

(ii) There is a disk $d^{\prime}(g)$ whose boundary intersects the boundary of $d(g)$ exactly in $d(g) \cap$ $d_{1}(g)$ and $d(g) \cap d_{2}(g)$, such that $c(g)$ bisects one of the two regions $d(g) \cap d^{\prime}(g)$ and $d(g) \cap \mathbb{R}^{2} \backslash d^{\prime}(g)$, which we call its lens region $\ell(g)$.

(iii) For any two edges $g_{1}$ and $g_{2}$ of $G_{i}$, the lens regions $\ell\left(g_{1}\right)$ and $\ell\left(g_{2}\right)$ are interior-disjoint.

(iv) The lens regions of the edges incident to the face in $D\left(G_{i}\right)$ corresponding to $m(g)$ cover the whole boundary of $d(g)$ and the endpoints of those regions appear in the same cyclic order as the according edges in $D\left(M_{i}\right)$.

Obviously, those invariants are fulfilled by $\Gamma_{p}$. Hence, assume that they are also fulfilled for $\Gamma_{i+1}$, and consider the removal of the edge $e=\left(v_{1}, v_{2}\right)$ from $M_{i+1}$ to obtain $M_{i}$. In the medial graph $G_{i+1}$, the edge $e$ corresponds to four edges sharing the vertex corresponding to $e$, and there are two unique faces corresponding to $v_{1}$ and $v_{2}$, respectively. Each of those has two of the edges of $G_{i+1}$ corresponding to $e$ as consecutive edges along the face. Let $g_{1}$ and $g_{2}$ be those consecutive incident edges on the face of $G_{i+1}$ corresponding to $v_{1}$. Note that their non-shared endpoints lie on the edges $\left(v_{1}, v_{3}\right)$ and $\left(v_{1}, v_{4}\right)$, respectively, where $v_{3}$ and $v_{4}$ are consecutive in the cyclic order around $v_{1}$ in $M_{i}$. Further, note that, when removing $e$ from $M_{i+1}$, we have to replace $g_{1}$ and $g_{2}$ by an edge $g$ connecting their non-shared endpoints. For every $j \in\{1,2,3,4\}$, let $d\left(v_{j}\right)$ be the disk of $C\left(M_{p}\right)$ that corresponds to the vertex $v_{j}$ of $M_{i} \subset M_{p}$ (note that with the notation from the invariants, $\left.d\left(v_{1}\right)=d\left(g_{1}\right)=d\left(g_{2}\right)\right)$. Next, consider $c\left(g_{1}\right)$ and $c\left(g_{2}\right)$ in the drawing $\Gamma_{i+1}$. By our invariants, $c\left(g_{1}\right)$ and $c\left(g_{2}\right)$ lie in their lens regions $\ell\left(g_{1}\right)$ and $\ell\left(g_{2}\right)$, which are consecutive along the boundary of $d\left(v_{1}\right)$. The only common point of $\ell\left(g_{1}\right)$ and $\ell\left(g_{2}\right)$ is the touching point of $d\left(v_{1}\right)$ and $d\left(v_{2}\right)$. The other endpoints of $c\left(g_{1}\right)$ and $c\left(g_{2}\right)$ are the touching points $d\left(v_{1}\right) \cap d\left(v_{3}\right)$ and $d\left(v_{1}\right) \cap d\left(v_{4}\right)$, respectively. Further, the boundary of $d\left(v_{1}\right)$ is completely covered by lens regions which are all pairwise non-intersecting and bounded by circles intersecting $\partial d\left(v_{1}\right)$ in right angles. We replace $c\left(g_{1}\right)$ and $c\left(g_{2}\right)$ by the circular arc $c(g)$ that has as its endpoints at the touching points $d\left(v_{1}\right) \cap d\left(v_{3}\right)$ and $d\left(v_{1}\right) \cap d\left(v_{4}\right)$ and is tangent to $c\left(g_{1}\right)$ and $c\left(g_{2}\right)$, respectively, in its endpoints. We define the lens region $\ell(g)$ as the unique region that contains $\ell\left(g_{1}\right)$ and $\ell\left(g_{2}\right)$ and is the intersection of $d\left(v_{1}\right)$ with the (according side of the) unique disk $d^{\prime}(g)$ for which $\partial d^{\prime}(g)$ intersects $\partial d\left(v_{1}\right)$ at a right angle in the endpoints of $c(g)$; see Figure 7.

Note that $\ell(g)$ does not intersect the interior of any other lens region: for the lens regions outside $d\left(v_{1}\right)$, this is trivial. For the ones inside $d\left(v_{1}\right)$, it follows from continuous transformation of the bounding circle $\partial d^{\prime}(g)$ to the bounding circle of the other lens. Hence, after repeating the analogous construction for the two other edges in $G_{i+1}$ needed to be replaced when removing $e$ from $M_{i+1}$, namely the ones that are incident to the face corresponding to $v_{2}$ in $D\left(G_{i+1}\right)$, we obtain a plane Lombardi drawing $\Gamma_{i}$ that again fulfills our four invariants, which completes the proof.

We remark that this result is not tight: there exist 4-regular plane multigraphs whose 


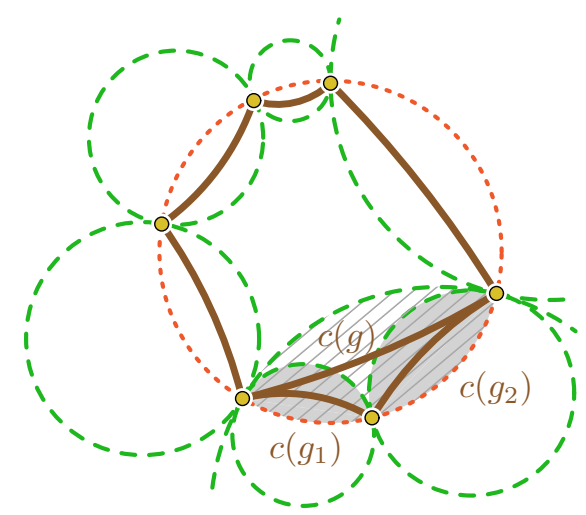

(a)

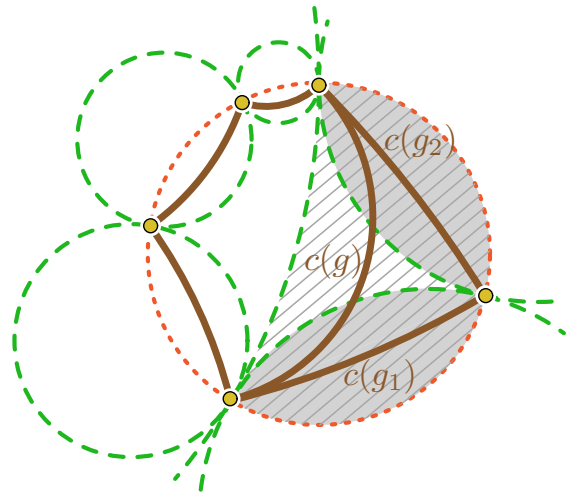

(b)

Figure 7: Two examples of a lens region $\ell(g)$ resulting from $\ell\left(g_{1}\right)$ and $\ell\left(g_{2}\right)$ : (a) convex and (b) reflex. The lens regions of $c\left(g_{1}\right)$ and $c\left(g_{2}\right)$ are drawn as shaded areas, while the one of $c(g)$ is the cross-hatched region.

primal-dual pair $M$ and $M^{\prime}$ contain parallel edges that still admit plane Lombardi drawings, e.g., knots $8_{12}, 8_{14}, 8_{15}, 8_{16}$; see Figure 26 in Appendix B.

Note that not all 4-regular graphs are medial graphs of a simple primal-dual pair (see, e.g. [15]), but we now prove that 4-regular polyhedral graphs are.

Lemma 6. Let $G=(V, E)$ be a 4-regular polyhedral graph and let $M$ and $M^{\prime}$ be the primaldual pair for which $G$ is the medial graph. Then $M$ and $M^{\prime}$ are simple.

Proof. Without loss of generality, assume for a contradiction that there are two edges between vertices $f$ and $g$ in $M$. Let $u$ and $v$ be the vertices of $G$ that these two edges pass through; see Figure 8. The vertices $f$ and $g$ of $M$ correspond to faces in the embedding of $G$ that both contain $u$ and $v$. Hence, the removal of $u$ and $v$ from $G$ disconnects $G$ into two parts: the part inside the area spanned by the two edges between $f$ and $g$ and the part outside this area. Both $u$ and $v$ have two edges in both areas, so either there is a multi-edge between $u$ and $v$, or there are vertices in both parts, which makes $u, v$ a separation pair of $G$. In either case, this contradicts the assumption that $G$ is polyhedral.

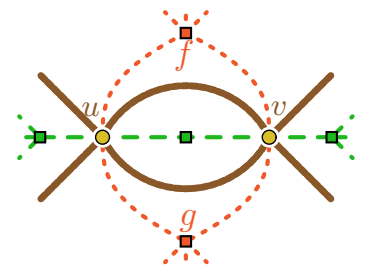

(a)

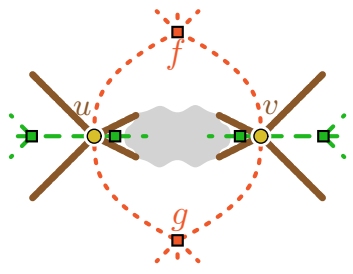

(b)

Figure 8: If there is a multi-edge between vertices $f$ and $g$ in the primal, then there is a multi-edge $(u, v)$ or a separation pair $u, v$ in the medial. 
Lemma 6 and Theorem 5 immediately give the following theorem.

Theorem 7. Let $G=(V, E)$ be a 4-regular polyhedral graph. Then $G$ admits a plane Lombardi drawing.

\section{Positive and Negative Results for Small Graphs}

We next consider all knots with 8 vertices or less. We compute plane Lombardi drawings for those that have it and argue that such drawings do not exists for the others. We start by showing that no knot with a $K_{4}$ subgraph is plane Lombardi.

Lemma 8. Every 4-regular plane multigraph $G$ that contains $K_{4}$ as a subgraph does not admit a plane Lombardi drawing.

Proof. Let $a, b, c, d$ be the vertices of the $K_{4}$. Every plane embedding of $K_{4}$ has a vertex that lies inside the cycle through the other 3 vertices; let $d$ be this vertex. Since $d$ has degree 4 , it has another edge to either one of $a, b, c$, or to a different vertex. In the former case, assume that there is a multi-edge between $c$ and $d$. In the latter case, by 4-regularity, there has to be another vertex of $a, b, c$ that is connected to a vertex inside the cycle through $a, b, c$; let $c$ be this vertex. In both cases, $c$ has two edges that lie inside the cycle through $a, b, c$. Further, again due to 4-regularity and planarity, the additional edges incident $a$ and $b$ go to the same face of the $K_{4}$, implying that one of the faces incident to $a b$ is empty.

Assume that $G$ has a Lombardi drawing. Since Möbius transformations do not change the properties of a Lombardi drawing, we may assume that the edge $(a, b)$ is drawn as a straight-line segment and that the face incident to $a b$ that contains the additional edges incident to $a$ and $b$ is the unbounded face; see Figure $9 \mathrm{~b}$. Since both $c$ and $d$ are neighbors of $a$ and $b$, there are two corresponding placement circles by Lemma 3. In fact, since any two edges of a Lombardi drawing of a 4-regular graph must enclose an angle of $90^{\circ}$ and since $a$ and $b$ have "aligned tangents" due to being neighbors themselves, the two placement circles coincide and a situation as shown in Figure $9 \mathrm{~b}$ arises. In particular, this means that in any vertex-minimal Lombardi drawing of $G$ the four vertices must be co-circular. Hence we cannot draw the missing circular arcs connecting $c$ and $d$ : any such arc must either lie completely inside or completely outside of the placement circle. Yet, the stubs for the two edges between $c$ and $d$ point inside at $c$ and outside at $d$.

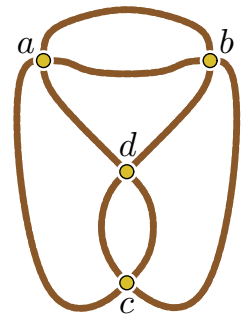

(a)

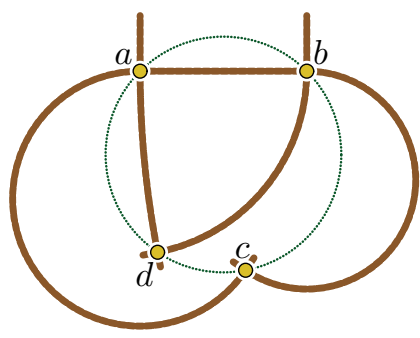

(b)

Figure 9: Knot $4_{1}$ has no Lombardi drawing. 


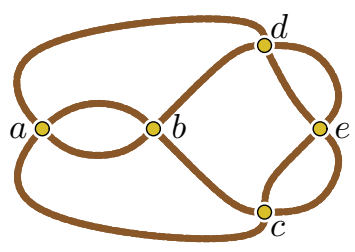

(a)

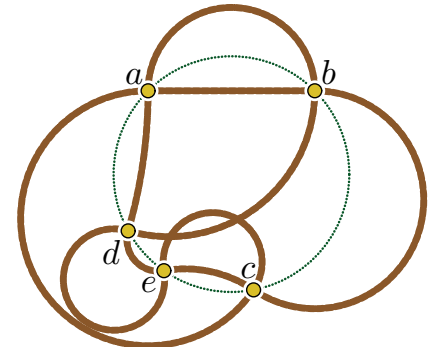

(b)

Figure 10: Knot $5_{2}$ and a non-plane Lombardi drawing.

Lemma 9. Knots $4_{1}$ and $5_{2}$ have no vertex-minimal Lombardi drawing.

Proof. Note that the combinatorial embeddings of vertex-minimal drawings for Knots $4_{1}$ and $5_{2}$ are unique up to the choice of the outer face [22]. Hence, as plane Lombardiness is invariant under Möbius transformations, either all of them or none of them have a plane Lombardi drawing.

For knot $4_{1}$, the claim immediately follows from Lemma 8.

Knot $5_{2}$ again has the property that all five vertices must be co-circular in any Lombardi drawing. To see this, we first consider the four vertices $a, b, c, d$ in Figure 10. Regardless of the placement of $a$ and $b$, we observe that $c$ and $d$ are both adjacent to $a$ and $b$ and need to enclose an angle of $90^{\circ}$ in the triangular face with $a$ and $b$. This situation was already discussed in Lemma 8 and yields a circle $C$ containing $a, b, c, d$; see Figure 9. The final vertex, $e$, is adjacent to $c$ and $d$ so that we can determine the placement circle for $e$ with respect to $c$ and $d$. As we know from Lemma 8, the two arc stubs of $d$ to be connected with $e$ form angles of $45^{\circ}$ with $C$ and point outwards. Conversely, the two arc stubs of $c$ form angles of $45^{\circ}$ with $C$ and point inwards. If we take any point $p$ on $C$ and draw circular arcs from the stubs of $c$ and $d$ to $p$, the four arcs meet at $90^{\circ}$ angles in $p$. These are precisely the angles required at vertex $e$ and hence $C$ is in fact the unique placement circle for $e$ by Lemma 3. This implies that actually all five vertices of $5_{2}$ must be co-circular in any Lombardi drawing.

Unlike knot $4_{1}$, it is geometrically possible to draw all edges as Lombardi arcs; see Figure 10b. However, as we will show, no plane Lombardi drawing of knot $5_{2}$ exists. By an appropriate Möbius transformation, we may assume that all five vertices are collinear on a circle of infinite radius. Moreover, to avoid crossings, the order along the line is either $a, b, c, e, d$ or $a, b, d, e, c$ (modulo cyclic shifts and reversals). Since both cases are symmetric, we restrict the discussion to the first one. As a further simplification, we initially assume that $a$ and $b$ are placed on the same position such that the lens between $a$ and $b$ collapses; see Figure 11.

This drawing consists of two intertwined 4-cycles, which intersect the line $\ell$ at angles of $45^{\circ}$. We argue that the 4 -cycle depicted in Figure 11 cannot be drawn as a simple cycle without self intersections. We consider the four centers $m_{1}, m_{2}, m_{3}, m_{4}$ of the circular arcs $a_{1}, a_{2}, a_{3}, a_{4}$ and their radii $r_{1}, r_{2}, r_{3}, r_{4}$. Due to the fact that adjacent arcs meet on $\ell$ at an 


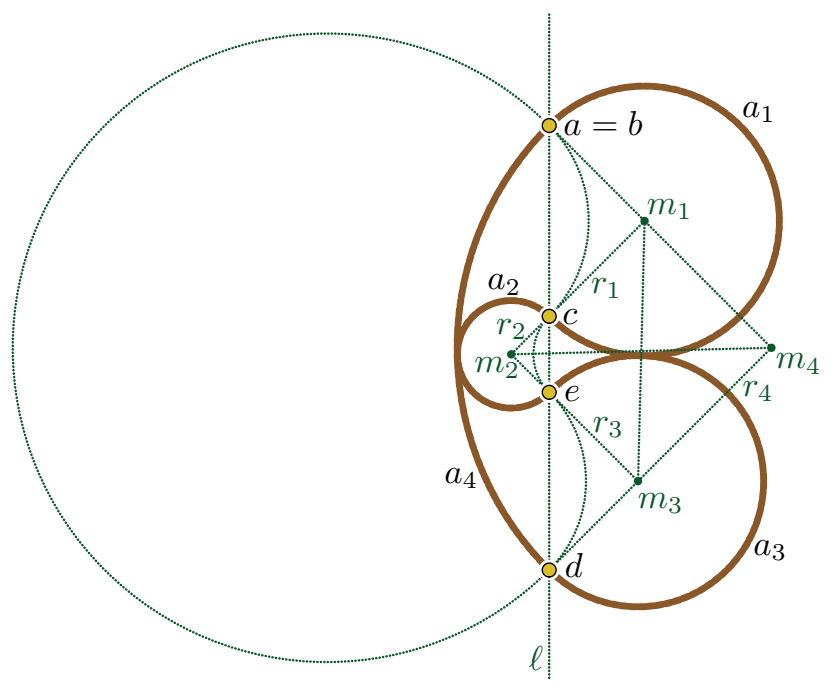

Figure 11: Knot $5_{2}$ has no plane Lombardi drawing.

angle of $45^{\circ}$ and have the same tangent, the four centers form the corners of a rectangle $R$ with side lengths $r_{1}+r_{2}$ and $r_{3}+r_{2}$. We can further derive that $r_{4}-r_{3}=r_{1}+r_{2}$. Let $\delta$ be the length of a diagonal of $R$. For the arcs $a_{1}$ and $a_{3}$ to be disjoint, we require $\delta>r_{1}+r_{3}$. For $a_{2}$ and $a_{4}$ to be disjoint, we require $\delta<r_{4}-r_{2}$. But since $r_{4}-r_{2}=r_{1}+r_{3}$, this is impossible and the 4-cycle must self-intersect.

Finally, if we move $b$ by some $\varepsilon>0$ away from $a$ and towards $c$, this will only decrease the radius $r_{4}$ and thus introduce proper intersections in the drawing. Thus, knot $5_{2}$ has no plane Lombardi drawing.

As the above lemma shows, even very small knots may not have a plane Lombardi drawing. However, most knots with a small number of crossings are indeed plane Lombardi. In Figure 26 in Appendix B, we provide plane Lombardi drawings of all knots with up to eight crossings except $4_{1}$ and $5_{2}$. Most of these drawings can actually be obtained using the techniques from Section 2 and 3.

Theorem 10. All knots with up to eight vertices other than $4_{1}$ and $5_{2}$ have a vertex-minimal plane Lombardi drawing.

Note that Theorem 10 implies that each of these knots has a combinatorial embedding that supports a plane Lombardi drawing. It is not true, however, that every embedding admits a plane Lombardi drawing. In fact, the knot $7_{5}$, as a member of an infinite family of knots and links, has an embedding that cannot be drawn plane Lombardi. This family is derived from the knot $5_{2}$ and gives rise to the next theorem.

Theorem 11. There exists an infinite family of prime knots and links that have vertexminimal non-Lombardi embeddings.

Proof. Consider again the knot $5_{2}$ (Figure 12a). By Lemma 9, it has no Lombardi drawing. If we duplicate the bottom vertex and vertically detach the two copies completely, then 


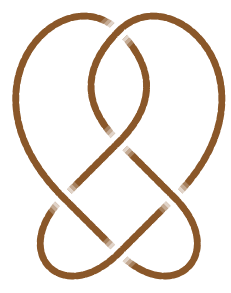

(a) $5_{2}$

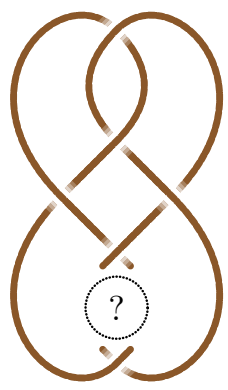

(b)

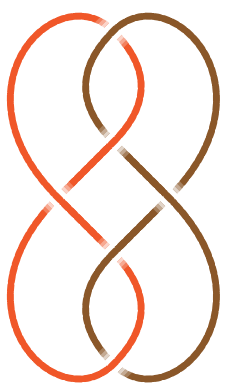

(c) $L_{6} a_{1}$

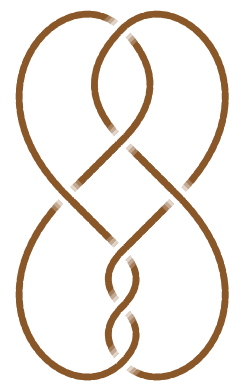

(d) $7_{5}$

Figure 12: A family of knots and links with vertex-minimal non-Lombardi embeddings.

the resulting graph (using four stubs to ensure the correct angular resolution) still has no plane Lombardi drawing (Figure 12b). As a result, we can construct an infinite family of graphs without plane Lombardi drawings by vertically twisting the connections between the duplicated vertices. After knot $5_{2}$, the next two members of this family are the link $L_{6} a_{1}$, consisting of two interlinked figure-8's (Figure 12c), and the knot $7_{5}$ (Figure 12d). For general $k \geq 2$, the graph obtained from replacing the crossing of knot $5_{2}$ by $k$ crossings is the prime knot or link that corresponds to the Conway notation $[k, 2,2]$; furthermore, the embeddings are alternating and reduced, so they are in fact vertex-minimal [2, Chapter 3.3].

However, the same family, starting with its six-vertex member $L_{6} a_{1}$, does have plane Lombardi drawings with a different embedding.

Corollary 12. The prime knots and links in the family of Theorem 11 with six or more vertices all have a vertex-minimal Lombardi embedding.

Proof. For the link $L_{6} a_{1}$ and the knot $7_{5}$ we provide plane Lombardi drawings in Figure 13. Observe that the incremental twists defining the family in Theorem 11 are now done at the bottom part of the knot/link diagrams in Figure 13. Since each twist now corresponds to a lens multiplication, we obtain from Lemma 4 that all other knots and links in the family also have plane Lombardi drawings. Figure 13 shows the respective 8-vertex link $L_{8} a_{11}$ and 9 -vertex knot 96 .

Interestingly, the two knots $4_{1}$ and $5_{2}$ without a plane Lombardi drawing belong to the known family of twist knots, which are knots formed by taking a closed loop, twisting it any number of times and then hooking up the two ends together. All other twist knots do have plane Lombardi drawings though.

Corollary 13. All twist knots except $4_{1}$ and $5_{2}$ have a vertex-minimal plane Lombardi drawing.

Proof. We know from Theorem 10 that $4_{1}$ and $5_{2}$ are not plane Lombardi knots. The smallest twist knot is $3_{1}$ and the other twist knots with at most eight vertices are $6_{1}, 7_{2}$, 


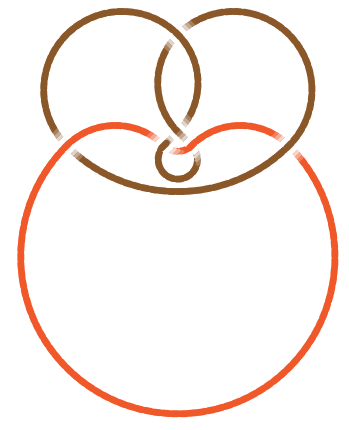

(a) $L_{6} a_{1}$

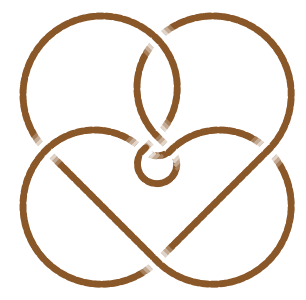

(b) $7_{5}$

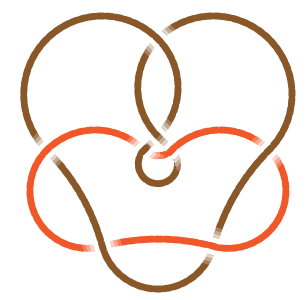

(c) $L_{8} a_{11}$

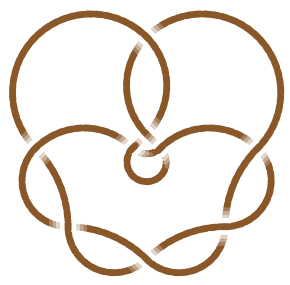

(d) 96

Figure 13: Plane Lombardi drawings for Corollary 12 obtained by lens multiplication.

and $8_{1}$, which all have a plane Lombardi drawing as shown in Figure 26. The progressive twisting pattern defining the twist knots and seen in the drawings of $6_{1}, 7_{2}$, and $8_{1}$ can easily be extended for all twist knots by incrementally applying the lens multiplication of Lemma 4. Since the considered drawings of the twist knots are alternating and reduced, these are in fact vertex-minimal diagrams of these knots [2, Chapter 3.3].

\section{Plane 2-Lombardi Drawings of Knots and Links}

Since not every knot admits a vertex-minimal plane Lombardi drawing, we now consider plane 2-Lombardi drawings; see Figure 14a for an example. Bekos et al [5] recently introduced smooth orthogonal drawings of complexity $k$. These are drawings where every edge consists of a sequence of at most $k$ circular arcs and axis-aligned segments that meet smoothly with horizontal or vertical tangents, and where at every vertex, each edge emanates either horizontally or vertically and no two edges emanate in the same direction. For the special case of 4-regular graphs, every smooth orthogonal drawing of complexity $k$ is also a plane $k$-Lombardi drawing. Alam et al. [3] showed that every plane graph with maximum degree 4 can be redrawn as a plane smooth-orthogonal drawing of complexity 2 . Their algorithm takes as input an orthogonal drawing produced by the algorithm of Liu et al. [17] and transforms it into a smooth orthogonal drawing of complexity 2. We show how to modify the algorithm by Liu et al., to compute an orthogonal drawing for a 4-regular plane multigraph and then use the algorithm by Alam et al. to transform it into a smooth orthogonal drawing of complexity 2 .

Theorem 14. Every biconnected 4-regular plane multigraph $G$ without loops admits a plane 2-Lombardi drawing with the same embedding.

Proof. The algorithm of Alam et al. [3] takes as input an orthogonal drawing produced by the algorithm of Liu et al. [17] and transforms it into a smooth orthogonal drawing of complexity 2. The drawings by Liu et al. have the property that every edge consists of at most 3 segments (except at most one edge that has 4 segments), and it contains no $S$-shapes, that is, it contains no edge that consists of 3 segments where the bends are in 


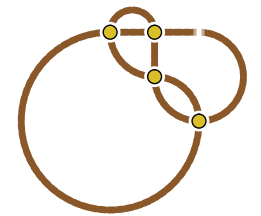

(a)

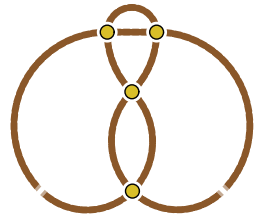

(b)

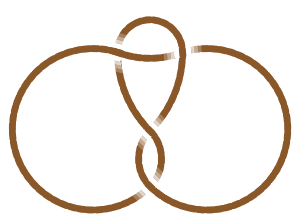

(c)

Figure 14: Drawings of knot 41 which by Lemma 9 does not admit a plane Lombardi drawing. (a) A smooth orthogonal drawing of complexity 2, (b) a different plane 2-Lombardi drawing, and (c) a plane $\varepsilon$-angle Lombardi drawing.

opposite direction. To show this theorem, we only have to show that we can apply the algorithm of Liu et al. to 4-regular plane multigraphs to produce a drawing with the same property.

Liu et al. first choose two vertices $s$ and $t$ and compute an st-order of the input graph. An $s t$-order is an ordering $(s=1,2, \ldots, n=t)$ of the vertices such that every $j$ $(2<j<n-1)$ has neighbors $i$ and $k$ with $i<j<k$. We can obtain an st-order for a multigraph by removing any duplicate edges. Liu et al. then direct all edges according to the $s t$-order from a vertex with lower st-number to a vertex with higher $s t$-number.

According to the rotation system implied by the embedding of the input graph, Liu et al. then assign a port to every edge around a vertex such that every vertex (except $t$ ) has an outgoing edge at the top port, every vertex (except $s$ ) has an incoming edge at the top port, every vertex has an outgoing edge at the right port if and only if it has at least 2 outgoing edges, and every vertex has an incoming edge at the left port if and only if it has at least 2 incoming edges. They further make sure the edge that uses the bottom port at $s$ is incident to the vertex $r$ with $s t$-number 2 , and that the edge $(s, t)$, if it exists, uses the left port at $s$ and the top port at $t$; this edge is the only one drawn with 4 segments, but can still be transformed into a smooth orthogonal edge of complexity 2 by Alam el al. . They place the vertices $s$ and $r$ on the $y$-coordinate 2 and every other vertex on the $y$-coordinates equal to their st-number. The shape of the edges is then implied by the assigned ports at their incident vertices. By placing vertices that share an edge with a bottom port and a top port above each other, there can be no S-shapes with two vertical segments, but there can still be S-shapes with two horizontal segments if an edge uses a left port and a right port. To eliminate these S-shapes, the consider sequences of S-shapes, that is, paths in the graphs that are drawn only with S-shapes, and move the vertices vertically such that they all lie on the same $y$-coordinate. Up to the elimination of S-shapes, every step of the algorithm can immediately applied to multigraphs. We choose $s$ and $t$ as vertices on the outer face of the given embedding such that the edge $(s, t)$ exists. We claim that then no multi-edge can be drawn as an S-shape.

Let $u$ and $v$ be two vertices in $G$ with at least two edges $e_{1}$ and $e_{2}$ between them. Without loss of generality, let $u$ have a lower st-number than $v$. Then both $e_{1}$ and $e_{2}$ are directed from $u$ to $v$. If $u=s$ and $v=r$, then both vertices are placed on the same $y$ coordinate, so there can be no S-shape between them. If $u=s$ and $v=t$, then there is an edge that uses the left port at $u$ and the top port at $v$; since all multi-edges have to be 
consecutive around $u$ and $v$, there can be no edge between them that uses a left port and a right port. Otherwise, assume that $e_{2}$ is the successor of $e_{1}$ in counter-clockwise order around $u$ (and hence the predecessor of $e_{1}$ in counter-clockwise order around $v$ ). If $e_{1}$ uses the right port at $u$ and the left port at $v$, then $e_{2}$ has to use the top port at $v$, which cannot occur by the port assignment. If $e_{1}$ uses the left port at $u$ and the right port at $v$, then $e_{2}$ has to use the bottom port at $u$, which also cannot occur by the port assignment. Thus, neither $e_{1}$ nor $e_{2}$ is drawn as an S-shape and every sequence of S-shapes consists only of simple edges. Hence, we can use the algorithm of Liu et al. to produce an orthogonal drawing with the desired property for every 4-regular plane multigraph and then use the algorithm of Alam et al. to transform it into a smooth complexity drawing of complexity 2 which is also a plane 2-Lombardi drawing.

Corollary 15. Every vertex-minimal embedding of a knot or link admits a plane 2-Lombardi drawing.

\section{Plane Near-Lombardi Drawings}

Since not all knots admit a vertex-minimal plane Lombardi drawing, in this section we relax the perfect angular resolution constraint. We say that an embedding of a knot (or a link) is near-Lombardi if it admits a drawing for every $\varepsilon>0$ such that

1. All edges are circular arcs,

2. Opposite edges at a vertex are tangent;

3. The angle between crossing pairs at each vertex is at least $90^{\circ}-\varepsilon$.

We call such a drawing a $\varepsilon$-angle Lombardi drawing. Note that a Lombardi drawing is equivalent to a 0 -angle Lombardi drawing. For example, the knot $4_{1}$ does not admit a vertexminimal plane Lombardi drawing, but it admits a vertex-minimal plane $\varepsilon$-angle Lombardi drawing, as depicted in Figure 14c.

Let $\Gamma$ be an $\varepsilon$-angle Lombardi drawing of a 4-regular graph. If each angle described by the tangents of adjacent circular arcs at a vertex in $\Gamma$ is exactly $90^{\circ}+\varepsilon$ or $90^{\circ}-\varepsilon$, then we call $\Gamma$ an $\varepsilon$-regular Lombardi drawing. Note that any Lombardi drawing is a 0-regular Lombardi drawing.

We first extend some of our results for plane Lombardi drawings to plane $\varepsilon$-angle Lombardi drawings. The following Lemma is a stronger version of Theorem 5.

Lemma 16. Let $G=(V, E)$ be a biconnected 4-regular plane multigraph and let $M$ and $M^{\prime}$ be the primal-dual multigraph pair for which $G$ is the medial graph. If one of $M$ and $M^{\prime}$ is simple, then $G$ admits a plane $\varepsilon$-regular Lombardi drawing preserving its embedding for every $0^{\circ} \leq \varepsilon<90^{\circ}$.

Proof. We use the same algorithm as for the proof of Theorem 5 with a slight modification. We first seek to direct the edges such that every vertex has two incoming opposite edges and two outgoing opposite edges. Let $M$ and $M^{\prime}$ be the primal-dual pair corresponding to the medial graph $G$. Every face in $G$ corresponds to a vertex either in $M$ or in $M^{\prime}$; we say that the face belongs to $M$ or $M^{\prime}$. We orient the edges around each face that belongs 


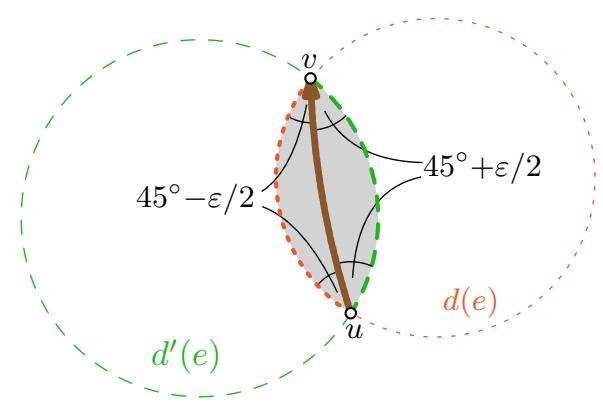

(a) Drawing a directed edge $e=(u, v)$ between the circles $d(e)$ and $d^{\prime}(e)$

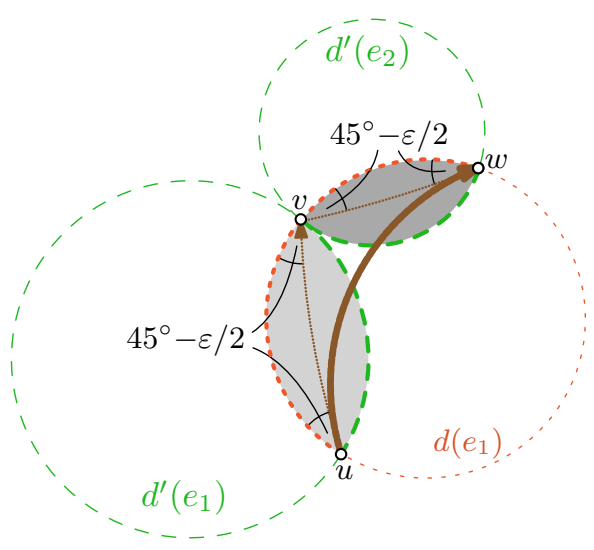

(b) Eliminating a vertex $v$ and adding the edge $(u, w)$ inside the circle $d\left(e_{1}\right)$

Figure 15: Illustrations for the proof of Lemma 16.

to $M$ in counter-clockwise order. Every edge in $G$ lies between a face that belongs to $M$ and a face that belongs to $M^{\prime}$, so this gives a unique orientation for every edge. Further, the faces around any vertex belong to $M$, to $M^{\prime}$, to $M$, and to $M^{\prime}$ in counter-clockwise order. Hence, the edges around any vertex are outgoing, incoming, outgoing, and incoming in counter-clockwise order, which gives us the wanted edge orientation.

We use the same primal-dual circle packing approach to obtain a drawing of $G^{\prime}$, but instead of using the bisection of the intersection of a primal and a dual circle, we use a circular arc with a different angle; see Figure 15a. Let $e=(u, v)$ be an edge of $G^{\prime}$ directed from $u$ to $v$, and let $l(e)$ be the lens region of $e$ between the primal-dual circles $d(e)$ and $d^{\prime}(e)$. Without loss of generality, assume that the $90^{\circ}$ angle inside $l(e)$ is between $d^{\prime}(e)$ and $d(e)$ in counter-clockwise order around $u$. In the proof of Theorem 5, we would draw $e$ as a bisection of $l(e)$. We draw $e$ that the angle between $d^{\prime}(e)$ and $(u, v)$ at $u$ is $45^{\circ}+\varepsilon / 2$ and the angle between $e$ and $d(e)$ at $u$ is $45^{\circ}-\varepsilon / 2$.

Informally, this means that all outgoing edges at a vertex are "rotated" by $\varepsilon / 2$ in counter-clockwise direction, and all incoming edges at a vertex are "rotated" by $\varepsilon / 2$ in clockwise direction compared to a plane circular-arc drawing of $G^{\prime}$. Since opposite edges of a vertex $u$ have the same direction with respect to $u$, they are rotated by the same angle, so they are still tangent. Further, since adjacent edges at $u$ have a different direction with respect to $u$, the angle between them is now either $90^{\circ}+\varepsilon$ or $90^{\circ}-\varepsilon$.

We then use the same procedure as in Theorem 5 to eliminate vertices from $G^{\prime}$ and obtain a plane $\varepsilon$-regular Lombardi drawing of $G$. In every step of this procedure, we eliminate a vertex $v$ from $G^{\prime}$ and add an edge between two pairs of its adjacent vertices (without introducing self-loops); see Figure 15b. Let $u$ and $w$ be two neighbors of $v$ in $G^{\prime}$ such that we want to obtain the edge $e=(u, w)$ in $G$. Without loss of generality, assume that the edge $e_{1}=(u, v)$ is directed from $u$ to $v$ in $G^{\prime}$ and that the edge $e_{2}=(v, w)$ is directed from $v$ to $w$ in $G^{\prime}$. Following the proof of Theorem $5, e_{1}$ lies in the lens region $l\left(e_{1}\right)$ between disks $d\left(e_{1}\right)$ and $d^{\prime}\left(e_{1}\right)$, and $e_{2}$ lies in the lens region $l\left(e_{1}\right)$ between disks $d\left(e_{2}\right)=d\left(e_{1}\right)$ 
and $d^{\prime}\left(e_{2}\right)$. Hence, $u$ and $w$ lie on a common circle $d\left(e_{1}\right)$ of the primal-dual circle packing. Assume that the $90^{\circ}$ angle inside $l\left(e_{1}\right)$ is between $d^{\prime}\left(e_{1}\right)$ and $d\left(e_{1}\right)$ in counter-clockwise order around $u$; the other case is symmetric. By the direction of the edges $e_{1}$ and $e_{2}$, the angle between $e_{1}$ and $d\left(e_{1}\right)$ is $45^{\circ}-\varepsilon / 2$ in counter-clockwise around $u$ and the angle between $d\left(e_{1}\right)$ and $e_{2}$ is also $45^{\circ}-\varepsilon / 2$ in counter-clockwise direction around $w$. Hence, we can draw the edge $e$ as a circular arc inside $d\left(e_{1}\right)$ with angle $45^{\circ}-\varepsilon / 2$ to $d\left(e_{1}\right)$ at both $u$ and $w$. We keep the ports at both vertices and by directing the edge from $u$ to $w$ we also keep a direction of the edges that satisfies the above property. Thus, we obtain a plane $\varepsilon$-regular Lombardi drawing of $G$.

The following Lemmas are stronger versions of Lemma 4 and Lemma 1, respectively. Since the proofs of the latter results do not rely on $90^{\circ}$ angles, they can also applied to the stronger versions. For the sake of completeness, a formal proof of Lemma 17 is still given.

Lemma 17. Let $G=(V, E)$ be a 4-regular plane multigraph with a plane $\varepsilon$-angle Lombardi drawing $\Gamma$. Then, any lens multiplication $G^{\prime}$ of $G$ also admits a plane $\varepsilon$-angle Lombardi drawing.

Proof. Let $f$ be a lens in $\Gamma$ spanned by two vertices $u$ and $v$. We denote the two edges bounding the lens as $e_{1}$ and $e_{2}$. Let $\alpha \in\left[90^{\circ}-\varepsilon, 90^{\circ}+\varepsilon\right]$ be the angle between $e_{1}$ and $e_{2}$ in both end-vertices. We define the bisecting circular arc $b$ of $f$ as the unique circular arc connecting $u$ and $v$ with an angle of $\alpha / 2$ to both $e_{1}$ and $e_{2}$. See Figure 4 for an example.

Let $p$ be the midpoint of $b$. If we draw circular $\operatorname{arcs} a_{1}$ and $a_{2}$ from both $u$ to $p$ and circular arcs $a_{3}$ and $a_{4}$ from $v$ to $p$ that have the same tangents as $e_{1}$ and $e_{2}$ in $u$ and $v$, then these four arcs meet at $p$ such that the angle between $a_{1}$ and $a_{2}$ as well as the angle between $a_{3}$ and $a_{4}$ is $\alpha$, whereas the angle between $a_{1}$ and $a_{4}$ and the angle between $a_{2}$ and $a_{3}$ is $180^{\circ}-\alpha \in\left[90^{\circ}-\varepsilon, 90^{\circ}+\varepsilon\right]$. Further, each such arc lies inside lens $f$ and hence does not cross any other arc of $\Gamma$. The resulting drawing is thus a plane $\varepsilon$-angle Lombardi drawing of a 4-regular multigraph that is derived from $G$ by subdividing the lens $f$ with a new degree- 4 vertex.

By repeating this construction inside the new lenses we can create plane $\varepsilon$-angle Lombardi drawings that replace lenses by chains of smaller lenses.

Lemma 18. Let $A$ and $B$ be two 4-regular plane multigraphs with plane $\varepsilon$-angle Lombardi drawings. Let $a$ be an edge of $A$ and $b$ an edge of $B$. Then the composition $A+B$ obtained by connecting $A$ and $B$ along edges $a$ and $b$ admits a plane $\varepsilon$-angle Lombardi drawing.

Let $G=(V, E)$ be a 4-regular plane multigraph and let $x \in V$ with edges $(x, a)$, $(x, b),(x, c)$, and $(x, d)$ in counter-clockwise order. A lens extension of $G$ is a 4-regular plane multigraph that is obtained by removing $x$ and its incident edges from $G$, and adding two vertices $u$ and $v$ to $G$ with two edges between $u$ and $v$ and the edges $(u, a),(u, b),(v, c),(v, d)$. Informally, that means that a vertex is substituted by a lens.

Lemma 19. Let $G=(V, E)$ be a 4-regular plane multigraph with a plane $\varepsilon$-angle Lombardi drawing $\Gamma$. Then, any lens extension of $G$ admits a plane $\left(\varepsilon+\varepsilon^{\prime}\right)$-angle Lombardi drawing for every $\varepsilon^{\prime}>0$. 


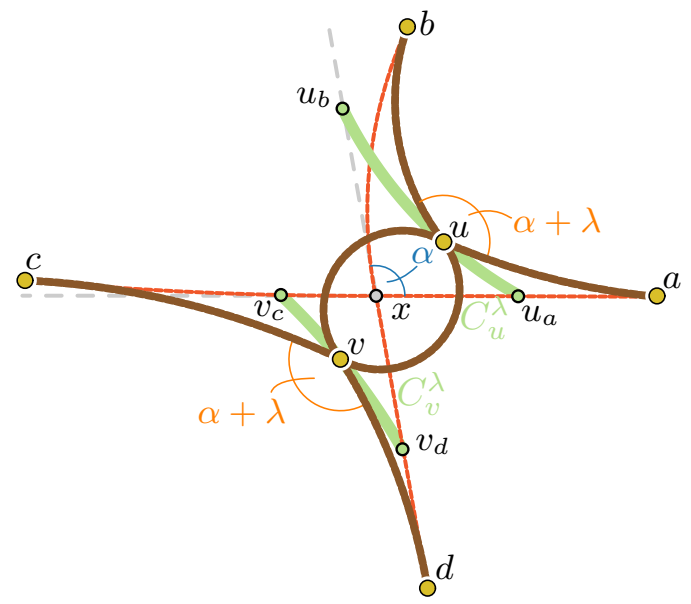

(a)

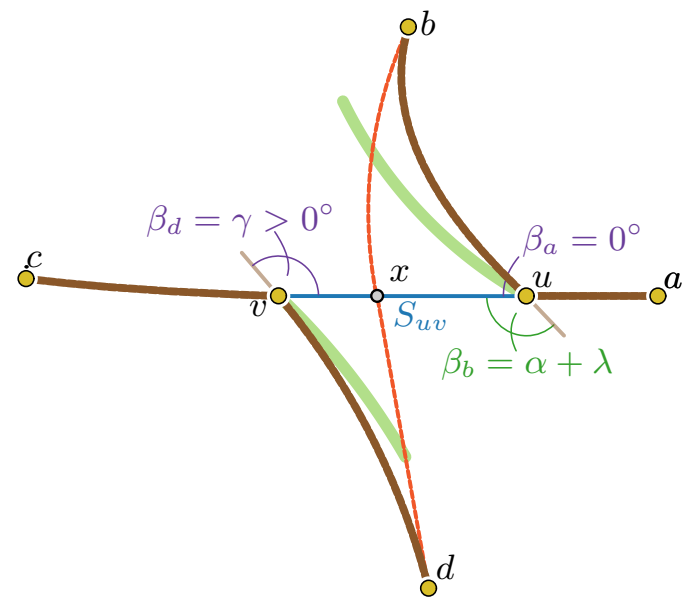

(b)

Figure 16: (a) The circular $\operatorname{arc} C_{u}^{\lambda}$ between $u_{a}$ and $u_{b}$ on the placement circles of $u$ and the circular arc $C_{v}^{\lambda}$ between $v_{c}$ and $v_{d}$ on the placement circles of $v$. (b) Placing $u$ on $u_{a}$ and $v$ on $v_{c}$ gives $\beta_{a}=0^{\circ}$.

Proof. Let $x \in V$ be the vertex that we want to perform the lens extension on such that we get the edges $(u, a),(u, b),(v, c),(v, d)$ in the obtained graph $G^{\prime}$. Let $\alpha$ be the angle between the tangents of $(x, a)$ and $(x, b)$ at $x$ in $\Gamma$. Since $\Gamma$ is a plane $\varepsilon$-angle Lombardi drawing, we have that $\alpha \leq 90^{\circ}+\varepsilon$. Further, the angle between the tangents of $(x, c)$ and $(x, d)$ at $x$ in $\Gamma$ is also $\alpha$, while the angles between the tangents of $(x, b)$ and $(x, c)$ at $x$ and between the tangents of $(x, d)$ and $(x, a)$ at $x$ are both $180^{\circ}-\alpha$. We apply the Möbiustransformation on $\Gamma$ that maps the edges $(x, a)$ and $(x, d)$ to straight-line segments and $a$ lies on the same $y$-coordinate and to the right of $x$; hence, $d$ lies strictly below $x$.

We aim to place $v$ such that the angle between the $\operatorname{arcs}(v, c)$ and $(v, d)$ is $\alpha+\lambda$ for some $0<\lambda \leq \varepsilon^{\prime}$ which we will show how to choose later. We have fixed ports at $c$ and $d$ and a fixed angle $\alpha+\lambda$ at $v$. According to Lemma 3, all possible positions of $v$ lie on a circle through $c$ and $d$. Note that the circle through $c, d, x$ describes all possible positions of neighbors of $c$ and $d$ with angle $\alpha$. Since the desired angle gets larger, the position circle for $v$ contains a point $v_{d}$ on the straight-line edge $(x, d)$ and a point $v_{c}$ on the half-line starting from $x$ with the angle of the port used by the arc $(x, c)$; see Figure 16a. We denote by $C_{v}^{\lambda}$ the circular arc between $v_{c}$ and $v_{d}$ on the placement circle of $v$ that gives the angle $\alpha+\lambda$ at $v$. We do the same construction for $u$ to obtain the circular $\operatorname{arc} C_{u}^{\lambda}$ between $u_{a}$ and $u_{b}$.

Since the drawing of $G$ is plane, there is some non-empty region in which we can move $x$ such that the $\operatorname{arcs}(x, a),(x, b),(x, c),(x, d)$ are drawn with the same ports at $a, b, c, d$ and do not cross any other edge of the drawing. We choose $\lambda$ as the largest value with $0<$ $\lambda \leq \varepsilon^{\prime}$ such that the two circular arcs $C_{u}$ and $C_{v}$ lie completely inside this region.

We now have to find a pair of points on $C_{v}^{\lambda}$ and $C_{u}^{\lambda}$ such that we can connect them via a lens. The ports of the two arcs we seek to draw between $u$ and $v$ lie opposite of the ports used by the arcs $(u, a),(u, b),(v, c)$, and $(v, d)$ We label the ports at $u$ and $v$ as $p_{u}^{a}$ opposite of $(u, a)$ at $u$, as $p_{u}^{b}$ opposite of $(u, b)$ at $u$, as $p_{v}^{c}$ opposite of $(v, c)$ at $v$, and 


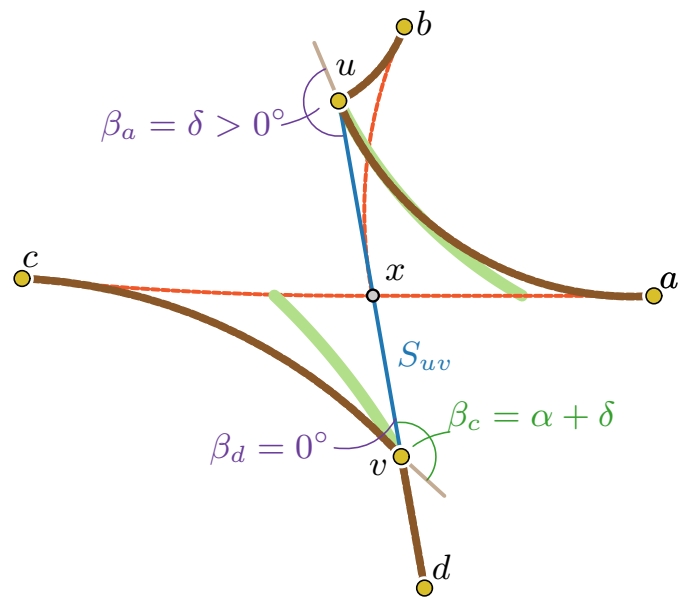

(a)

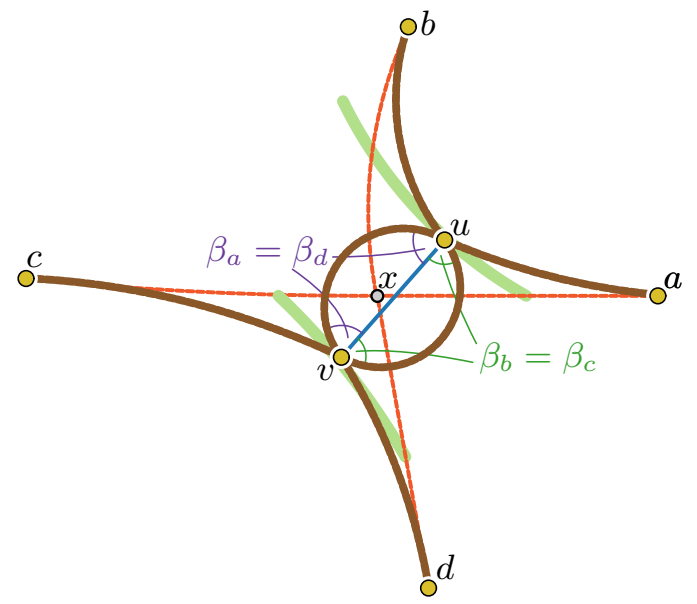

(b)

Figure 17: (a) Placing $u$ on $u_{b}$ and $v$ on $v_{d}$ gives $\beta_{d}=0^{\circ}$. (b) Placing $u$ and $v$ such that $\beta_{a}=\beta_{d}$ gives a lens between $u$ and $v$ with the desired angles.

as $p_{v}^{d}$ opposite of $(v, d)$ at $v$. We have to find a pair of points on $C_{u}^{\lambda}$ and $C_{v}^{\lambda}$ such that these ports are "compatible": Take a point $q_{u}$ on $C_{u}^{\lambda}$ and a point $q_{v}$ on $C_{v}^{\lambda}$ and connect them by a segment $S_{u v}$. Then the angle $\beta_{b}$ between $S_{u v}$ and $p_{u}^{b}$ has to be the same as the angle $\beta_{c}$ between $S_{u v}$ and $p_{v}^{c}$, and the angle $\beta_{a}$ between $S_{u v}$ and $p_{u}^{a}$ has to be the same as the angle $\beta_{d}$ between $S_{u v}$ and $p_{v}^{d}$. By construction, we have that $\beta_{a}+\beta_{b}=90^{\circ}+\alpha+\lambda$ and $\beta_{c}+\beta_{d}=90^{\circ}+\alpha+\lambda$, so it suffices to find a pair of points such that $\beta_{a}=\beta_{d}$.

Assume that $v$ is placed on $v_{c}$ and $u$ is placed on $u_{a}$; see Figure 16b. The edge $(x, a)$ is drawn as a straight-line segment, and the edge $(x, c)$ uses the port opposite of the one of $(x, a)$. Hence, the segment $S_{u v}$ is a segment through $x$. Furthermore, it uses exactly the port $p_{u}^{a}$ at $u$, so we have $\beta_{a}=0^{\circ}$. On the other hand, $\beta_{d}$ is strictly positive: The segment $(x, d)$ enters $x$ with an angle of $\gamma=180^{\circ}-\alpha>0^{\circ}$ to the segment $(x, v)$. Since $v$ lies to the left of $x$, the angle described between the tangent of the circular arc $(v, d)$ at $v$ and the segment $(v, x)$ is strictly larger than $\gamma$. Since $\beta_{d}$ is described by the same tangent and segment, we have that $\beta_{d}=\gamma>0^{\circ}$.

Now assume that $v$ is placed on $v_{d}$ and $u$ is placed on $u_{b}$; see Figure 17a. The edge $(x, d)$ is drawn as a straight-line segment, and the edge $(x, b)$ uses the port opposite of the one of $(x, d)$. Hence, the segment $S_{u v}$ is a segment through $x$. Furthermore, it uses exactly the port $p_{u}^{d}$ at $u$, so we have $\beta_{d}=0^{\circ}$. On the other hand, $\beta_{a}$ is strictly positive: The segment $(x, a)$ enters $x$ with an angle of $\delta=90^{\circ}+\alpha>0^{\circ}$ to the segment $(x, v)$. Since $u$ lies above $x$, the angle described between the tangent of the circular arc $(u, a)$ at $u$ and the segment $(u, x)$ is strictly larger than $\delta$. Since $\beta_{d}$ is described by the same tangent and segment, we have that $\beta_{d}=\delta>0^{\circ}$.

Hence, we have found a pair of points for $u$ and $v$ such that $\beta_{a}=0^{\circ}$ and $\beta_{d}=\gamma>0^{\circ}$ and we have found a pair of points for $u$ and $v$ such that $\beta_{a}=\delta>0^{\circ}$ and $\beta_{d}=0 \circ$. Since we can move $u$ and $v$ freely along the curves $C_{u}$ and $C_{v}$ between these pairs of points, $\beta_{a}$ can become any angle between $0^{\circ}$ and $\delta$ and $\beta_{d}$ can become any angle between $0^{\circ}$ and $\gamma$. 


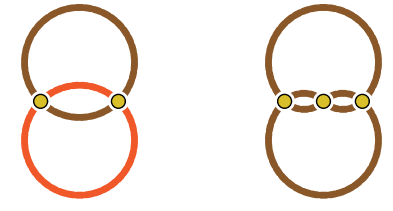

(a)
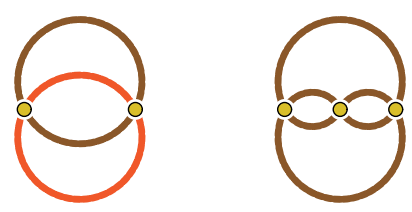

(b)

Figure 18: The only biconnected 4-regular multigraphs with at most 3 vertices. (a) plane Lombardi and (b) plane $\varepsilon$-angle Lombardi drawings.

Thus, there has to exist some pair of points for $u$ and $v$ such that $\beta_{a}=\beta_{d}$; see Figure 17b. We choose this pair of points and connect $u$ and $v$ by two circular arcs such that one of them uses the ports $p_{u}^{a}$ and $p_{v}^{d}$ and the other one uses the ports $p_{u}^{b}$ and $p_{v}^{c}$. Note that the $\operatorname{arcs}(u, a)$ and $(u, b)$ are now drawn the same way as if we moved $x$ onto the determined position of $u$ and the $\operatorname{arcs}(v, c)$ and $(v, d)$ are now drawn the same way as if we moved $x$ onto the determined position of $v$. Hence, by the choice of $\lambda$, they do not introduce any crossing and thus the drawing is plane.

Lemma 20. Every 4-regular plane multigraph with at most 3 vertices admits a plane $\varepsilon$ regular Lombardi drawing for every $0 \leq \varepsilon<90^{\circ}$.

Proof. There are only two 4-regular multigraphs with at most 3 vertices and each of them has a plane Lombardi drawing as depicted in Figure 18a. For some $0^{\circ}<\varepsilon<90^{\circ}$, we can obtain a plane $\varepsilon$-regular Lombardi drawing by simply making the circular arcs larger or smaller, as depicted in Figure 18b.

We are now ready to present the main result of this section. The proof boils down to a large case distinction using the tools developed in the previous discussion. We split the original graph into biconnected components and then use Lemma 20 and 16 as base cases. With the help of lens extensions, lens multiplications, and knot sums we can combine the "near-Lombardi" drawings of the biconnected graphs to generate an "near-Lombardi" drawing of the original graph. As a consequence, every knot is near-Lombardi.

Theorem 21. Let $G=(V, E)$ be a biconnected 4-regular plane multigraph wihtout loops and let $\varepsilon>0$. Then $G$ admits a plane $\varepsilon$-angle Lombardi drawing.

Proof. If $G$ has at most 3 vertices, then we obtain a plane Lombardi drawing of $G$ by Lemma 20. So assume that $G$ is a biconnected 4-regular plane graph with $n \geq 4$. We seek to draw $G$ by recursively by splitting it into smaller graphs. We prove our algorithm by induction on the number of vertices; to this end, suppose that every biconnected 4-regular plane graph with at most $n-1$ vertices admits a plane $\varepsilon^{\prime}$-angle Lombardi drawing for every $\varepsilon^{\prime}>0$; this holds initially for $n=4$. We proceed as follows.

Case 1. $G$ is polyhedral. In this case, we can draw it plane Lombardi using Theorem 5.

Case 2. $G$ contains a multilens, that is, a sequence of lenses between the vertices $u_{1}, \ldots, u_{k}$ with $k \geq 2$. We contract the lenses to a single lens, that is, we remove the vertices $u_{2}, \ldots, u_{k-1}$ 


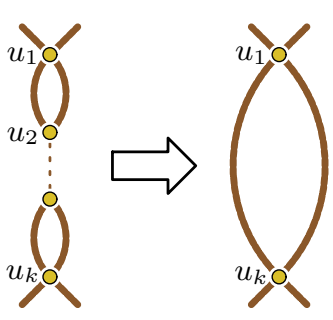

(a) Case 2

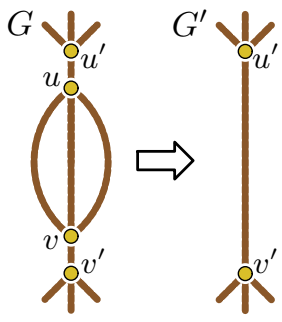

(b) Case 3.2

Figure 19: Illustrations for the proof of Theorem 21.

and their incident edges from $G$ and add two edges between $u_{1}$ and $u_{k}$ to form a new graph $G^{\prime}$; see Figure 19a. This operation is essentially a reverse lens multiplication and introduces no self-loops.

Case 3. $G$ contains a lens between two vertices $u$ and $v$, but it contains no multilens. We consider three subcases based on the number of edges between $u$ and $v$ in $G$.

Case 3.1. There are four edges between $u$ and $v$ in $G$. Since $G$ is 4-regular, it consists exactly of these two vertices and four edges and can be drawn by Lemma 20 .

Case 3.2. There are three edges between $u$ and $v$ in $G$; see Figure 19b. Then there exists also some edge $\left(u, u^{\prime}\right)$ and some edge $\left(v, v^{\prime}\right)$ in $G$. Since $G$ is biconnected, we have $u^{\prime} \neq v^{\prime}$; otherwise, it would be a cutvertex. We remove $u$ and $v$ from $G$ and add an edge between $u^{\prime}$ and $v^{\prime}$ to form a new graph $G^{\prime}$. This operation preserves biconnectivity as $u^{\prime}$ and $v^{\prime}$ form a separation pair in $G$ and it introduces no self-loops because $v \neq v^{\prime}$. Hence, the graph $G^{\prime}$ is a biconnected 4-regular plane graph with $n-2$ vertices and by induction admits a plane $\varepsilon$-angle Lombardi drawing. Let $G^{\prime \prime}$ be the graph that consists of $u$ and $v$ and four multiedges between them. This graph has a plane $\varepsilon$-regular Lombardi drawing by Lemma 20. Furthermore, $G$ can be obtained by adding $G^{\prime}$ and $G^{\prime \prime}$ along the edge $\left(u^{\prime}, v^{\prime}\right)$ of $G^{\prime}$ and one of the edges of $G^{\prime \prime}$. Using Lemma 18 , we can obtain a plane $\varepsilon$-angle Lombardi drawing of $G$.

Case 3.3. There are two edges between $u$ and $v$ in $G$. We consider two subcases.

Case 3.3.1. Removal of $u$ and $v$ from $G$ preserves connectivity; see Figure 20a. We contract $u$ and $v$ to a new vertex: we remove them from $G$ and add a new vertex $x$ that is connected to the neighbors of $u$ and $v$ different from $u$ and $v$ to form $G^{\prime}$. This operation preserves biconnectivity: Since $G$ is biconnected, the only cutvertex in $G^{\prime}$ can be $x$; but since the removal of $u$ and $v$ from $G$ preserves connectivity, so does the merged vertex $x$. Since there are exactly two edges between $u$ and $v$, the new vertex $x$ has degree 4 . Hence, $G^{\prime}$ is a biconnected 4-regular plane graph with $n-1$ vertices and by induction admits a plane $\varepsilon / 2$-angle Lombardi drawing. Furthermore, $G$ can be obtained from $G^{\prime}$ by a lens extension on $x$. We obtain a plane $\varepsilon$-angle Lombardi drawing of $G$ using Lemma 19.

Case 3.3.2. The removal of $u$ and $v$ from $G$ disconnects the graph, that is, $u$ and $v$ form a separation pair in $G$; see Figure 20b. Since there are exactly two edges between $u$ and $v$, their removal disconnects $G$ into two connected components $A$ and $B$ with at least two vertices each (otherwise, there would be a self-loop). Furthermore, $G$ contains an edge from $u$ to a vertex $u_{A}$ in $A$ and another edge from $u$ to a vertex $u_{B}$ in $B$. If this would not 


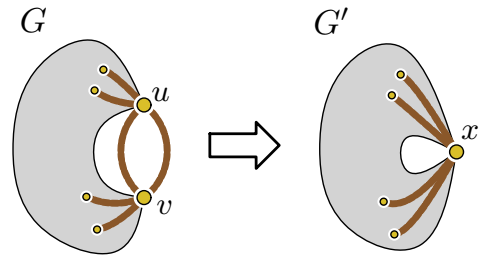

(a) Case 3.3.1

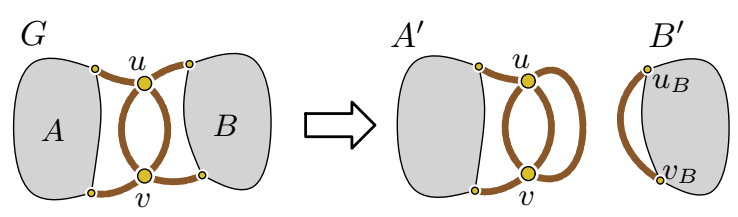

(b) Case 3.3.2

Figure 20: Illustrations for Case 3.3 in the proof of Theorem 21.

be the case than $v$ would be a cutvertex in $G$. Analogously, there is an edge from $v$ to a vertex $v_{A}$ in $A$ and an edge from $v$ to a vertex $v_{B}$ in $B$. We have $u_{A} \neq v_{A}$ (and $\left.u_{B} \neq v_{B}\right)$; otherwise, this vertex would be a cutvertex in $G$.

Let $A^{\prime}$ be the graph $G-B$ with an additional edge between $u$ and $v$. Since $G$ is biconnected, there are two disjoint paths in $G$ between any two vertices from $A$. Only one of these paths can "leave" $A$ through the separation pair $u, v$. Hence, we can redirect the part outside $A$ to the new edge $(u, v)$ in $A^{\prime}$, which shows that every two vertices in $A^{\prime}$ are connected with at least two disjoint paths. This shows that $A^{\prime}$ is biconnected.

Let $B^{\prime}$ be the graph $B$ with an additional edge between $u_{B}$ and $v_{B}$. We can show that $B^{\prime}$ is biconnected by the same arguments we have applied for $A^{\prime}$ : In $G$ there have to be two disjoint paths between every vertex pair from $B$. Only one of these paths can leave $B$ over the separation pair $u, v$ and this part can be replaced by the new edge that we added to $B^{\prime}$. Hence between every two vertices in $B^{\prime}$ we have two disjoint paths, which proves that $B^{\prime}$ is a biconnected 4 -regular plane graph with at most $n-4$ vertices. By induction $B^{\prime}$ admits a plane $\varepsilon$-angle Lombardi drawing. Furthermore, $G$ can be obtained by adding $A^{\prime}$ and $B^{\prime}$ along one of the edges between $u$ and $v$ of $A^{\prime}$ and the edge $\left(u_{B}, v_{B}\right)$ of $B^{\prime}$. Using Lemma 18 , we can obtain a plane $\varepsilon$-angle Lombardi drawing of $G$.

Case 4. $G$ is simple, but not 3 -connected, so there exists at least one separation pair that splits $G$ into at least two connected components. Let $A_{u, v}$ be a smallest connected component induced by the separation pair $u, v$. We say that $u, v$ is a minimal separation pair if $A_{u, v}$ does not contain any separation pair and there is no separation pair between a vertex of $A_{u, v}$ and either $u$ or $v$.

We create two biconnected 4-regular plane plane graphs as follows; see Figure 21a. Let $A$ be the subgraph of $G$ induced by the vertices in $A_{u, v}, u$, and $v$, let $B$ be the subgraph of $G$ that contains all vertices not in $A_{u, v}$ and all edges not in $A$; in particular, there is no edge $(u, v)$ in $B$. By this construction, all edges of $G$ are either part of $A$ or part of $B$ and both $A$ and $B$ are connected, and every vertex is part of either $A$ or $B$, except the two vertices $u$ and $v$ which are part of both. However, $A$ and $B$ are not 4-regular, so we create two 4-regular graphs $A^{\prime}$ and $B^{\prime}$ for the recursion as follows. Let $\operatorname{deg}_{A}(u), \operatorname{deg}_{A}(v), \operatorname{deg}_{B}(u), \operatorname{deg}_{B}(v)$ be the degree of $u$ and $v$ in $A$ and $B$, respectively, with $\operatorname{deg}_{A}(u)+\operatorname{deg}_{B}(u)=\operatorname{deg}_{A}(v)+\operatorname{deg}_{B}(v)=4$.

Case 4.1. $\operatorname{deg}_{A}(u)=1$ or $\operatorname{deg}_{A}(v)=1$. Without loss of generality, let $\operatorname{deg}_{A}(u)=1$. Let $x$ be the neighbor of $u$ in $A$. We have that $x \neq v$ since otherwise $A$ consists only of a single 


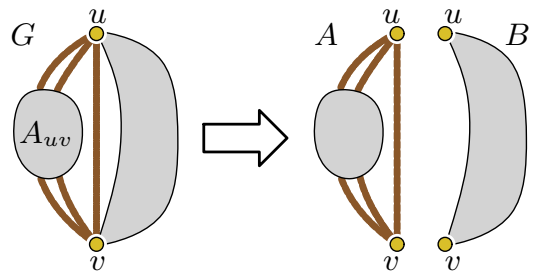

(a) Case 4

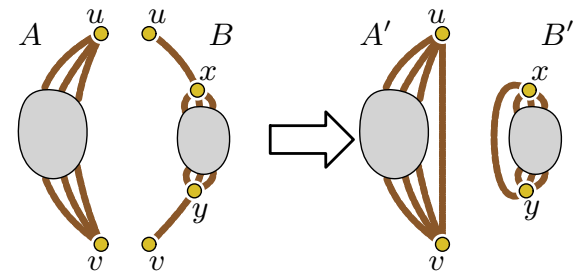

(b) Case 4.2

Figure 21: Illustrations for Case 4 in the proof of Theorem 21.

edge (if $\operatorname{deg}_{A}(v)=1$ ) or $v$ is a cutvertex in $G$ (if $\operatorname{deg}_{A}(v)=3$ ). Then $x, v$ is a separation pair of $G$ whose removal gives a connected component $A_{x, v}$ with less vertices than $A_{u, v}$, as it contains the same vertices but not $x$, contradicting the minimality of the separation pair $u, v$.

Case 4.2. $\operatorname{deg}_{A}(u)=\operatorname{deg}_{A}(v)=3$; see Figure 21b. We add an edge between $u$ and $v$ to $A$ to obtain the graph $A^{\prime}$. The resulting graph is biconnected: consider any pair of vertices $a, b \in A^{\prime}$. There were at least two vertex-disjoint paths in $G$ between $a$ and $b$. Since $u, v$ is a separation pair in $G$, at most one of these two paths traverses vertices in $G-A$, and any path through these vertices must contain $u$ and $v$. Hence, there is a path that traverses the same edges in $A^{\prime}$ and uses the newly introduced edge between $u$ and $v$ instead.

We remove $u$ and $v$ from $B$ and add an edge between their neighbors to form $B^{\prime}$. Let $x$ be the neighbor of $u$ in $B$ and let $y$ be the neighbor of $v$ in $B$. We have that $x \neq y$ since otherwise $x$ would be a cutvertex in $G$. Hence, we introduce no self-loops. With a similar argument, $B^{\prime}$ is also biconnected, as any path between two vertices through vertices in $A$ has to traverse $u$ and $v$ and-since they both have degree 1 in $B$ - their neighbors, so the path can use the newly introduced edge instead.

We recursively obtain a plane $\varepsilon$-angle Lombardi drawing of $A^{\prime}$ and $B^{\prime}$. Since both $A^{\prime}$ and $B^{\prime}$ have fewer vertices than $G$, they admit one by induction. To obtain a drawing of $G$ from $A^{\prime}$ and $B^{\prime}$, we have to remove the edge $(u, v)$ from $A^{\prime}$ and the edge $(x, y)$ from $B^{\prime}$ and we have to add the edges $(u, x)$ and $(v, y)$. This procedure is equivalent to adding $A^{\prime}$ and $B^{\prime}$ along these respective edges, so we can solve it using the algorithm described in Lemma 18.

Case 4.3. $\operatorname{deg}_{A}(u)=\operatorname{deg}_{A}(v)=2$. We consider two more subcases.

Case 4.3.1. The separation pair $u, v$ splits $G$ into three connected components $A_{u, v}, B_{u, v}$, and $C_{u, v}$; see Figure 22. We add two edges between $u$ and $v$ to $A$ to obtain $A^{\prime}$. Let $u_{B}$ be the neighbor of $u$ in $B_{u, v}$ and let $v_{B}$ be the neighbor of $v$ in $B_{u, v}$. We have that $u_{B} \neq v_{B}$, as otherwise it would be a cutvertex of $G$. We obtain the 4-regular multigraph $B^{\prime}$ by adding an edge between $u_{B}$ and $v_{B}$ to $B_{u, v}$. By the same argument as in Case 3.3.2, $B^{\prime}$ is biconnected. Analogously, we obtain the biconnected 4-regular multigraph $C^{\prime}$ by adding an edge between the neighbor $u_{C}$ of $u$ in $C_{u, v}$ and the neighbor $v_{C}$ of $v$ in $C_{u, v}$ to $C_{u, v}$. We recursively create a plane $\varepsilon$-angle Lombardi drawing of $A^{\prime}, B^{\prime}$, and $C^{\prime}$. Then, we create a plane $\varepsilon$-angle Lombardi drawing with the use of Lemma 18 by adding $A^{\prime}$ and $B$ along one edge between $u$ and $v$ of $A^{\prime}$ and the edge $\left(u_{B}, v_{B}\right)$ of $B$, and adding the resulting graph and $C$ along the other edge between $u$ and $v$ and the edge $\left(u_{C}, v_{C}\right)$ of $C$. 


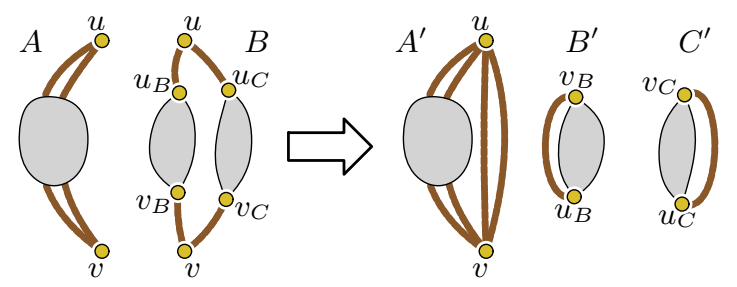

Figure 22: Illustration for Case 4.3.1 in the proof of Theorem 21.

Case 4.3.2. The separation pair $u, v$ splits $G$ into two connected components $A_{u, v}$ and $B_{u, v}$; see Figure 23. In this case, the graph $B$ consists of $B_{u, v}, u$, and $v$ and the edges incident to $u$ or $v$ and a vertex of $B_{u, v}$.

We add two edges between $u$ and $v$ to both graphs $A$ and $B$ to obtain $A^{\prime}$ and $B^{\prime}$. Let $M$ and $M^{\prime}$ be the primal-dual pair for which $A^{\prime}$ is the medial graph. We claim that $M$ or $M^{\prime}$ is simple. Let $u_{1}$ and $u_{2}$ be the neighbors of $u$ in $A$ and let $v_{1}$ and $v_{2}$ be the neighbors of $v$ in $A$. Since $G$ is simple, there is no multi-edge between $u$ and $v$ in $A$. Furthermore, there is no single edge $(u, v)$ in $A$, since otherwise $u$ and $v$ would each only have one neighbor in $A_{u, v}$ and these neighbors would be a separation pair of $G$ that induces a smaller connected component. Thus, each of $u_{1}, u_{2}, v_{1}, v_{2}$ is different from $u$ and $v$ and we introduce no selfloops. By construction, the graph $A_{u, v}$ contains no separation pair and thus has either at most 3 vertices or is 3 -connected. We claim that $A^{\prime}$ is 3 -connected. If $A_{u, v}$ has only 1 vertex, then $u_{1}=u_{2}$, so there is a multi-edge in $A_{u, v}$ which contradicts simplicity of $G$. If $A_{u, v}$ has only 2 vertices, then there has to be a multi-edge between them, which again contradicts simplicity of $G$. If $A_{u, v}$ has only 3 vertices, then there have to be 4 edges in $A_{u, v}$, which also contradicts simplicity of $G$. If $A_{u, v}$ has at least 4 vertices, then $u$ and $v$ have at least 3 different neighbors in $A_{u, v}$, as otherwise there would be a cutvertex or a separation pair that gives a smaller connected component than the separation pair $u, v$. Thus, if $u$ and $v$ are connected to at least 3 vertices of $A_{u, v}$ and $u$ and $v$ are connected by an edge, which preserves 3 -connectivity. Hence, $A^{\prime}$ is 3 -connected. Since $G$ is simple, there is no multi-edge between $u$ and $v$ in $A$. Furthermore, there is no single edge $(u, v)$ in $A$, since otherwise $u$ and $v$ would each only have one neighbor in $A_{u, v}$ and these neighbors would be a separation pair of $G$ that induces a smaller connected component. Hence, $A^{\prime}$ has no separation pair and exactly one multi-edge between $u$ and $v$. By Lemma 6 , that means that $M$ and $M^{\prime}$ have exactly one pair of parallel edges in total, so one of them has to be simple.

We recursively obtain a plane $\varepsilon$-angle Lombardi drawing of $B^{\prime}$. Let $90^{\circ}+\alpha$ be the angle described by the tangents of the two edges between $u$ and $v$ at $u$. Note that $\alpha$ might be negative, but $|\alpha| \leq \varepsilon$. Since the primal or dual of $A^{\prime}$ is simple, we obtain a plane $|\alpha|$-regular Lombardi drawing of $A^{\prime}$ by Lemma 16. Thus, the angle described by the tangents of the two edges between $u$ and $v$ at $u$ is either $90^{\circ}+\alpha$ or $90^{\circ}-\alpha$. We can make sure that the angle is $90^{\circ}+\alpha$ by inverting the direction of all edges in the proof of Lemma 16 in case it is not.

We perform a Möbius-transformation on the drawing of $A^{\prime}$ such that the edges between $u$ and $v$ are drawn with an angle of $45^{\circ}+\alpha / 2$ between either edge and the segment between $u$ and $v$. We pick the Möbius-transformation such that $u$ and $v$ are very close to 


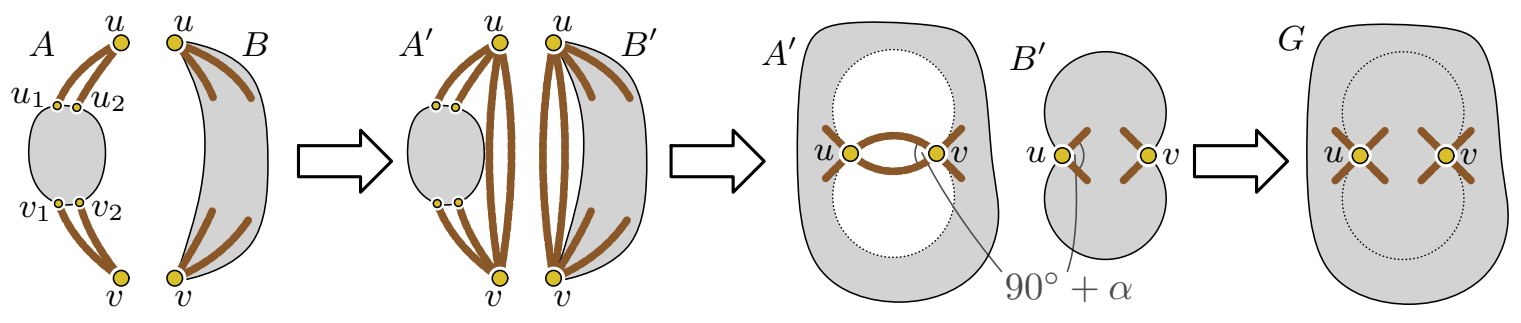

Figure 23: Illustration for Case 4.3.2 in the proof of Theorem 21.

each other; in particular, we want them to be close enough such that the two circles that the edges between $u$ and $v$ lie on contain no other vertex of $A^{\prime}$ and no edges of $A^{\prime}$ that is incident to neither $u$ nor $v$. Note that the radius of these circles are the same and approach 0 as the distance between $u$ and $v$ approaches 0 ; hence, such a Möbius-transformation exists.

We apply another Möbius-transformation on $B^{\prime}$ such that the distance between $u$ and $v$ is the same as in the drawing of $A^{\prime}$ and such that the two edges between $u$ and $v$ are drawn with an angle of $135^{\circ}-\alpha / 2$ between either edge and the segment between $u$ and $v$. We now place the drawing of $B^{\prime}$ on the drawing of $A^{\prime}$ such that both copies of $u$ lie on the same coordinate and both copies of $v$ lie on the same coordinate and then we remove all edges between $u$ and $v$. By construction, the whole drawing of $B^{\prime}$ lies inside the region described by the two edges between $u$ and $v$ in the drawing of $B^{\prime}$. Further, since these edges lie on the same circles as the two edges between $u$ and $v$ in $A^{\prime}$, this region contains no vertices or edges in the drawing of $A^{\prime}$ (except $u$ and $v$ and their incident edges themselves). Since the drawings of $A^{\prime}$ and $B^{\prime}$ are plane and we cannot introduce a crossing between an edge of $A^{\prime}$ and an edge of $B^{\prime}$ after removing the multi-edges between $u$ and $v$, the resulting drawing of $G$ is also plane. Since $u$ and $v$ use the same ports in the drawing of $A^{\prime}$ and the drawing of $B^{\prime}$, the resulting drawing is a plane $|\alpha|$-angle Lombardi drawing of $G$. Because of $|\alpha| \leq \varepsilon$, this drawing is also a plane $\varepsilon$-angle Lombardi drawing of $G$.

Corollary 22. Every vertex-minimal embedding of a knot or link admits a plane $\varepsilon$-angle Lombardi drawing.

\section{Conclusion and Open Problems}

We have studied plane Lombardi drawings of knots and links, which can be modeled as 4-regular multigraphs. We have shown that not all knots admit a crossing-minimal plane Lombardi drawing. On the other hand, we have given an algorithm to draw 4-regular polyhedral graphs plane Lombardi. Further, we have shown that every biconnected 4-regular plane multigraph admits a plane 2-Lombardi drawing, where every edge is composed of two circular arcs, and a plane near-Lombardi drawing, where the angle between two edges at a vertex is at least $90^{\circ}-\varepsilon$ for any $\varepsilon>0$, while the angle between opposite edges remains $180^{\circ}$.

Although we made progress on the original question, there are several questions that remain open. As main questions concerning Lombardi drawings we have the following. 
Question 1. Can we give a complete characterization of 4-regular plane multigraphs that admit a plane Lombardi drawing?

Question 2. What is the complexity of deciding whether a given 4-regular plane multigraph admits a plane Lombardi drawing?

Question 3. Given a 4-regular plane multigraph, what is the minimum number of edges consisting of two circular arcs in any plane 2-Lombardi drawing?

Acknowledgements. Research for this work was initiated at Dagstuhl Seminar 17072 Applications of Topology to the Analysis of 1-Dimensional Objects which took place in February 2017. We thank Benjamin Burton for bringing the problem to our attention, Dylan Thurston for helpful discussion, and the anonymous reviewer for useful comments.

\section{References}

[1] Knot drawing in Knot Atlas. http://katlas.org/wiki/Printable_Manual\#Drawing_ Planar_Diagrams. Accessed: 2017-06-08.

[2] Colin C. Adams. The Knot Book: An Elementary Introduction to the Mathematical Theory of Knots. American Mathematical Society, 2004. URL: https://bookstore. ams.org/knot/.

[3] Muhammad Jawaherul Alam, Michael A. Bekos, Michael Kaufmann, Philipp Kindermann, Stephen G. Kobourov, and Alexander Wolff. Smooth orthogonal drawings of planar graphs. In Alberto Pardo and Alfredo Viola, editors, Proc. 13th Lat. Am. Symp. Theoret. Inform. (LATIN'14), number 8392 in Lecture Notes Comput. Sci., pages 144155. Springer, 2014. doi:10.1007/978-3-642-54423-1_13.

[4] J. W. Alexander and G. B. Briggs. On types of knotted curves. Ann. Math., 28:562-586, 1927. doi:10.2307/1968399.

[5] Michael A. Bekos, Michael Kaufmann, Stephen G. Kobourov, and Antonios Symvonis. Smooth orthogonal layouts. J. Graph Algorithms Appl., 17(5):575-595, 2013. doi: $10.7155 /$ jgaa. 00305 .

[6] Graham R. Brightwell and Edward R. Scheinerman. Representations of planar graphs. SIAM J. Discrete Math., 6(2):214-229, 1993. doi:10.1137/0406017.

[7] Peter R. Cromwell. Arc presentations of knots and links. Banach Center Publ., 42:5764, 1998. URL: https://eudml .org/doc/208825.

[8] Peter R Cromwell. Knots and links. Cambridge University Press, 2004. doi:10.1017/ CB09780511809767.

[9] Hubert de Fraysseix and Patrice Ossona de Mendez. On a characterization of Gauss codes. Discrete Comput. Geom., 22(2):287-295, 1999. doi:10.1007/PL00009461. 
[10] C. H. Dowker and Morwen B. Thistlethwaite. Classification of knot projections. Topol. Appl., 16(1):19-31, 1983. doi:10.1016/0166-8641 (83)90004-4.

[11] Christian A. Duncan, David Eppstein, Michael T. Goodrich, Stephen G. Kobourov, Maarten Löffler, and Martin Nöllenburg. Planar and poly-arc Lombardi drawings. $J$. Comput. Geom., 9(1):328-355, 2018. doi:10.20382/jocg.v9i1a11.

[12] Christian A. Duncan, David Eppstein, Michael T. Goodrich, Stephen G. Kobourov, and Martin Nöllenburg. Lombardi drawings of graphs. J. Graph Algorithms Appl., 16(1):37-83, 2012. doi:10.7155/jgaa.00251.

[13] David Eppstein. Planar Lombardi drawings for subcubic graphs. In W. Didimo and M. Patrignani, editors, Proc. 20th Int. Symp. Graph Drawing (GD'12), volume 7704 of Lecture Notes Comput. Sci., pages 126-137. Springer, 2013. doi:10.1007/ 978-3-642-36763-2_12.

[14] David Eppstein. A Möbius-invariant power diagram and its applications to soap bubbles and planar Lombardi drawing. Discrete Comput. Geom., 52:515-550, 2014. doi:10. $1007 /$ s00454-014-9627-0.

[15] David Eppstein and Michael B. Dillencourt. Uninscribable 4-regular polyhedron. Electronic Geometry Models p. 2003.08.001, 2003. URL: http://www.eg-models.de/ models/Polytopes/2003.08.001.

[16] Louis H. Kauffman. On Knots, volume 115 of Ann. Math. Stud. Princeton University Press, 1987. URL: https://press .princeton.edu/titles/2587.html.

[17] Yanpei Liu, Aurora Morgana, and Bruno Simeone. A linear algorithm for 2-bend embeddings of planar graphs in the two-dimensional grid. Discrete Appl. Math., 81(13):69-91, 1998. doi:10.1016/S0166-218X (97)00076-0.

[18] Charles Livingston. Knot Theory, volume 24 of Carus Math. Monogr. Mathematical Association of America, 1993. doi:10.5948/UP09781614440239.

[19] Dale Rolfsen. Knots and Links. American Mathematical Soc., 1976. URL: https: //bookstore.ams.org/chel-346-h/.

[20] Robert G. Scharein. Interactive Topological Drawing. PhD thesis, Department of Computer Science, The University of British Columbia, 1998. URL: https://knotplot. $\mathrm{com} /$ thesis/.

[21] H. Schubert. Die eindeutige Zerlegbarkeit eines Knotens in Primknoten. Sitzungsber. Heidelberger Akad. Wiss., Math.-naturwiss. Klasse, 3. Abh., 1949. doi:10.1007/ 978-3-642-45813-2.

[22] Peter Guthrie Tait. On knots. Trans. Roy. Soc. Edinburgh, 28:145-190, 1879. URL: https://www.maths.ed.ac.uk/ v1ranick/papers/taitonknots.pdf.

[23] William T. Tutte. How to draw a graph. Proc. London Math. Soc, 13(3):743-768, 1963. doi:10.1112/plms/s3-13.1.743. 
A A Full Example for Drawing Knots $5_{1}, 6_{2}, 7_{7}$, and $8_{18}$ via Circle Packing
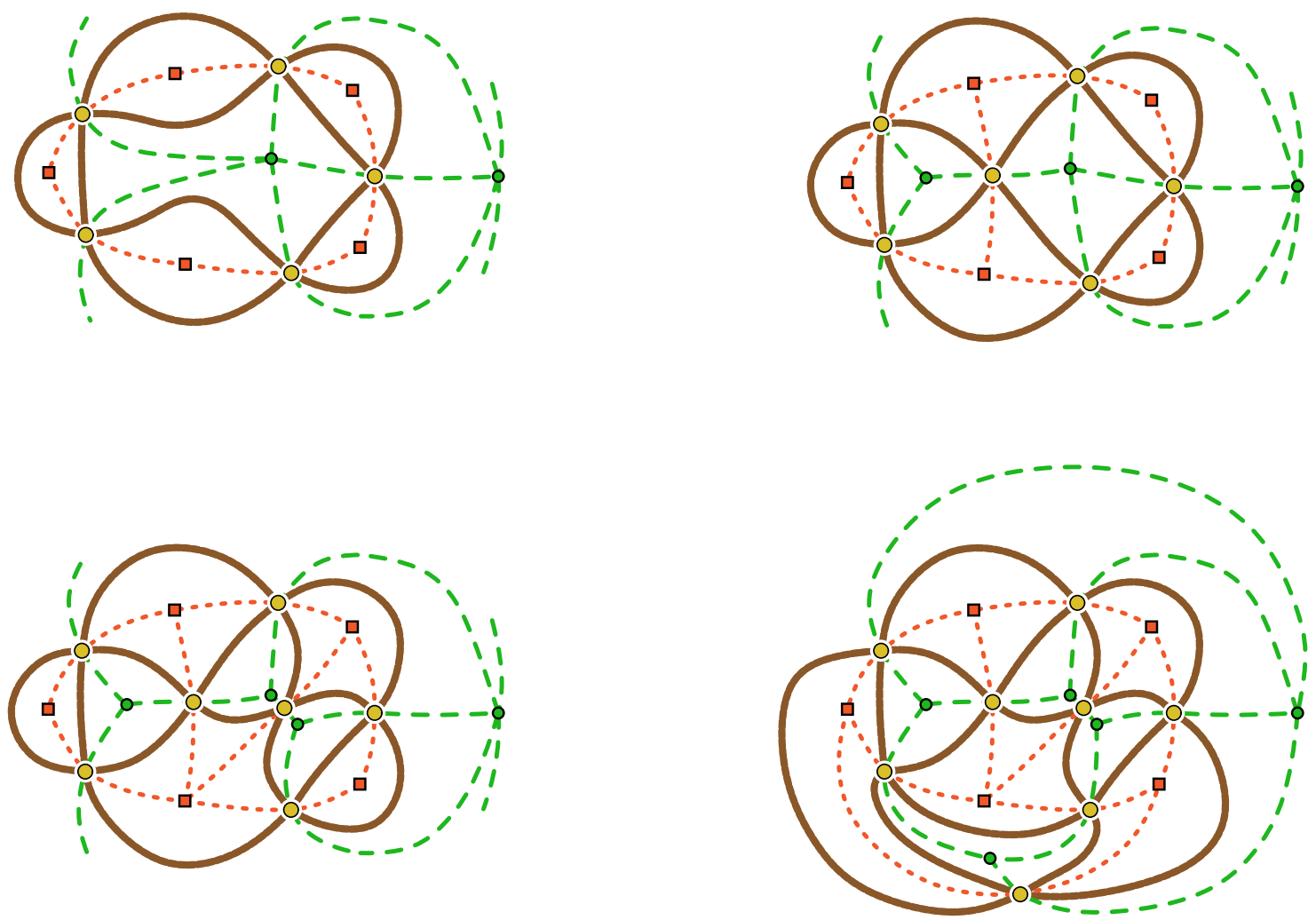

Figure 24: Extension of the primal graph (dotted) of knot $5_{1}$ to the square pyramid and its dual (dashed). The medial graph in the top right is the knot $6_{2}$, the medial graph in the bottom left is the knot $7_{7}$, and the medial graph in the bottom right is the knot $8_{18}$. 
(a)

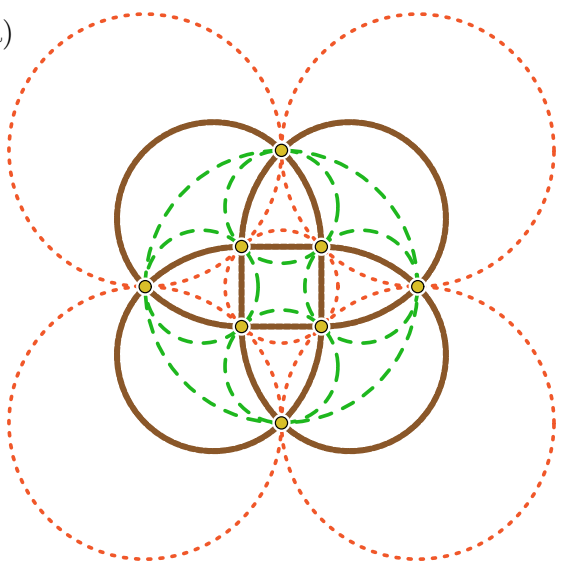

(c)

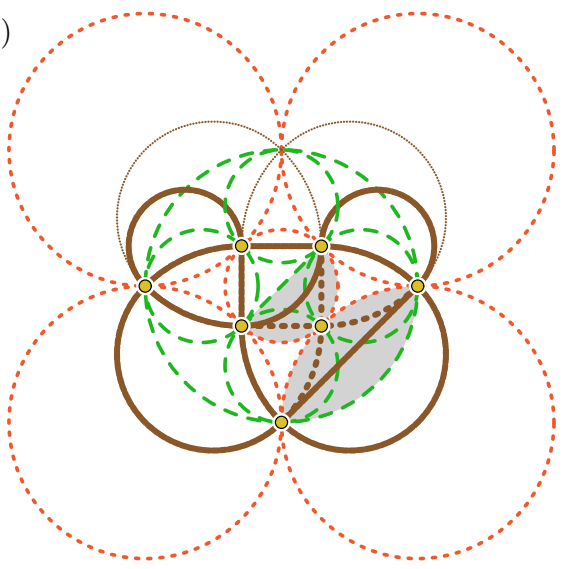

(e)

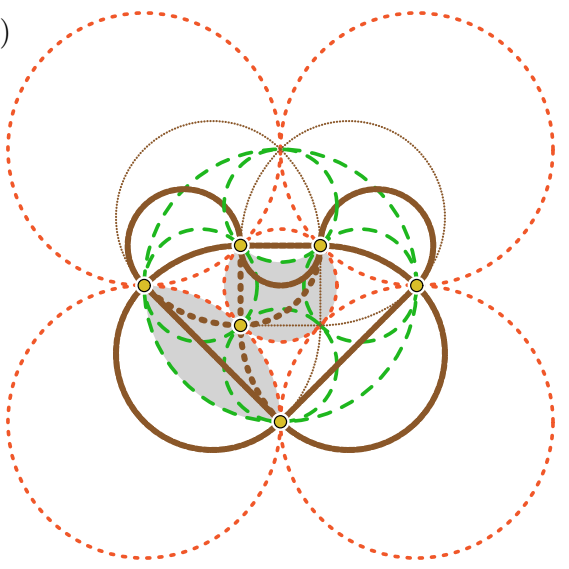

(b)

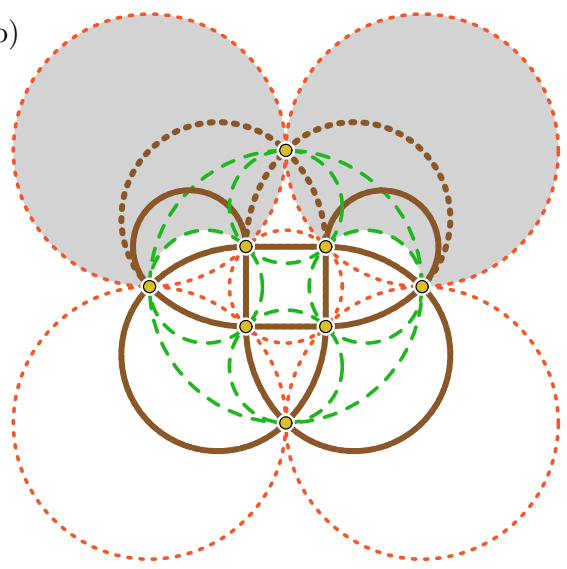

(d)

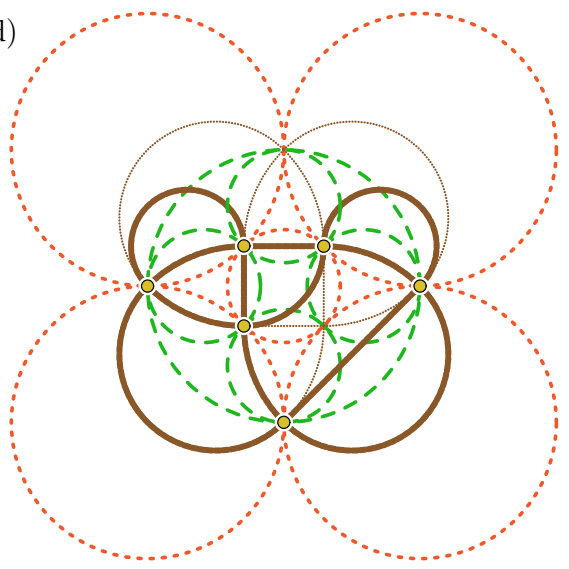

(f)

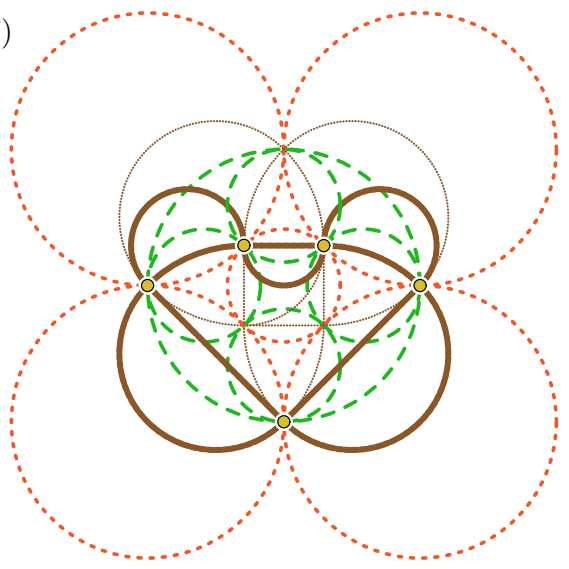

Figure 25: (a) A circle packing for the square pyramid (dotted) and its dual (dashed), and a plane Lombardi drawing for the medial graph $8_{18}$ (solid); (b) eliminating an edge of the primal and the plane Lombardi drawing of $7_{7}$; (c) eliminating an edge of the primal; (d) the plane Lombardi drawing of $6_{2}$; (e) eliminating an edge of the primal; (f) the plane Lombardi drawing of $5_{1}$. 


\section{B Drawings of all Lombardi Knots up to 8 Vertices}
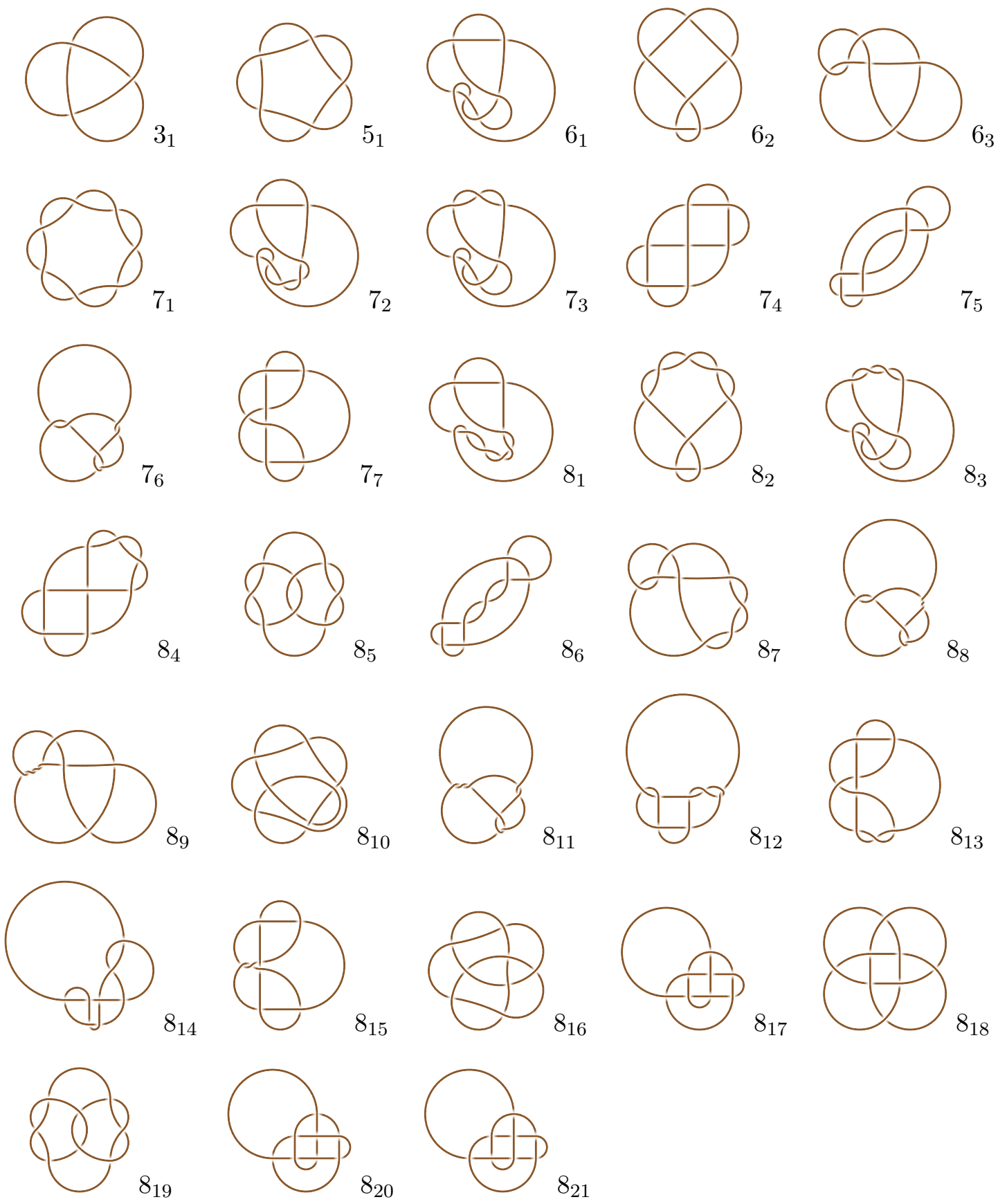

Figure 26: Plane Lombardi drawings of all Lombardi knots up to 8 vertices. 